\title{
ALGEBRAIC COSET CONFORMAL FIELD THEORIES
}

\author{
FENG XU
}

\begin{abstract}
All unitary Rational Conformal Field Theories (RCFT) are conjectured to be related to unitary coset Conformal Field Theories, i.e., gauged Wess-ZuminoWitten (WZW) models with compact gauge groups. In this paper we use subfactor theory and ideas of algebraic quantum field theory to approach coset Conformal Field Theories. Two conjectures are formulated and their consequences are discussed. Some results are presented which prove the conjectures in special cases. In particular, one of the results states that a class of representations of coset $W_{N}(N \geq 3)$ algebras with critical parameters are irreducible, and under the natural compositions (Connes' fusion), they generate a finite dimensional fusion ring whose structure constants are completely determined, thus proving a long-standing conjecture about the representations of these algebras .
\end{abstract}

\section{§1. INTRODUCTION}

Conformal field theories (CFT) in two dimensions (cf. [MS]) have attracted great attention among mathematicians in recent years. A large class of CFT known as Rational CFT (RCFT) are more amenable than general CFT and the classification of RCFT is an outstanding open problem.

Unitary coset CFT is a gauged Wess-Zumino-Witten (WZW) model with a compact Lie group $G$ as a gauge group, and $H$, a subgroup of $G$, is gauged (cf. [KS]). It has been conjectured (cf. [MS], [Witten]) that all unitary RCFT (except perhaps orbifolds, which are relatively better understood and also similar to the coset CFT, cf. Page 428 of [MS]) are related to unitary coset CFT. In the literature there has been several different mathematical approaches to CFT, see, for an example, [DL] for an approach by using vertex operator algebras (cf. [B] or [FLM]). However, in the case of WZW model with a compact gauge group $G$, there is a manifestly unitary formulation of these unitary CFT by using subfactor theory (cf. [J], [W1] and [L1]) and ideas from algebraic quantum field theory (cf. [Haag]). This formulation has various advantages besides producing the expected results, see, for an example, $[\mathrm{X} 1]$ and $[\mathrm{X} 2]$ for some results on certain rings which seem to be invisible in other approaches. In view of the importance of coset CFT among all RCFT, it is natural

I'd like to thank the referee for helpful suggestions. This work is partially supported by NSF grant DMS-9820935. 1991 Mathematics Subject Classification. 46S99, 81R10.

Typeset by $\mathcal{A} \mathcal{M} \mathcal{S}-\mathrm{T}_{\mathrm{E}} \mathrm{X}$ 
to use this unitary formulation to study unitary coset CFT, and this is the main purpose of this paper.

To illustrate the new ideas in this paper we will focus on the case when $G$ is a simply connected semisimple compact Lie group of type $A$, i.e., $G=S U\left(N_{1}\right) \times$ $S U\left(N_{2}\right) \times \ldots \times S U\left(N_{n}\right)$. The ideas of this paper can be applied to all compact semisimple and simply connected Lie groups and we plan to consider them in separate publications.

We will describe in more details about the paper. In subsection 2.1 , We define conformal precosheaf and their covariant representations as in [GL]. We then show how coset $H \subset G$ naturally gives rise to an irreducible conformal precosheaf (Prop. 2.2) together with a class of covariant representations. As a first step we proved in Th. 2.3 an irreducibility result in our setting by using vertex operators (cf. [FZ] or $[\mathrm{Kacv}])$. To do this we show in Prop. 2.3 that certain smeared vertex operators are affiliated (cf. $[\mathrm{Mv}]$ ) with some von Neumann algebras. Note that these smeared vertex operators are generally unbounded operators and one must be careful with the formal manuplations of them. Prop. 2.3 is proved by using a series of lemmas (lemma 1-6). It can be seen from the proof that the vertex operator algebra for coset $H \subset G$ as defined on P. 67 of [Kacv] or $\S 5$ of [FZ] can be thought as "germs" of our irreducible conformal precosheaf.

Based on physicists' argument and well known examples, in subsection 2.3 two conjectures are formulated about these representations. The first conjecture states that the representations generate a finite dimensional ring under certain compositions, in other words, the CFT is really "rational". The second conjecture is a formula about the square root of the minimal index or the statistical dimension (cf. 4.1) of these representations in terms of certain limits of characters, referred to as "Kac-Wakimoto" formula in [L5]. Both conjectures are highly nontrivial. In particular, the second conjecture implies Kac-Wakimoto conjecture in $\S 2$ of $[\mathrm{KW}]$.

The rest of the paper is devoted to the proof of the two conjectures in special cases.

Section 3 is about proving finite index by using certain commuting squares which play an important role in the type $I I_{1}$ subfactor theory (cf. [Po], [We] ). However, we will consider factors of type $I I I_{1}$. First we introduced a notion of co-finiteness for a pair $H \subset G$. This notion is motivated by the conjectures of 2.3. Prop. 3.1, which follows from the commuting squares in lemma 3.1, is a key observation of this paper. It implies that if $H_{1} \subset H_{2} \subset G$ and $H_{1} \subset G$ is cofinite, then $H_{1} \subset H_{2}$ is cofinite. This leads to an infinite series of cofinite pairs in Cor. 3.1.

Section 4 can be considered as an application of [X1] and [X2] given the results of 3.1. We first recall some of the results of [X1] which are used in 4.2 to determine certain ring structures. These results are summarized in Th. 4.1. Prop. 4.2 is the key observation in section 4. It allows one to study the representations of coset $H \subset G$ by using the theory which has been developed for $G$ and $H$ in some cases. Then we prove Th. 4.2 which states that for a pair $H \subset G$ which is cofinite, if similar results in Chap. V of [W2] hold for $H$, then Conj. 1 is also true. This result and Cor. 3.1 imply Conj. 1 for the infinite series in Cor. 4.2. 
In 4.3 we study the cosets corresponding to diagonal inclusions by using Th. 4.2 and prove in Th. 4.3 that Conj. 2 is true for these cosets. We also prove in Th. 4.3 certain irreducibility result.

The results in Th. 4.3 in the special case of coset $W_{N}$ algebras with critical parameters have been long anticipated in both mathematics and physics literature. For example these results are related to the conjectures in $\S 3$ and 4 of [FKW], which are conjectured for what is know as "W-algebras", and they are closely related to coset $W$-algebras in the vertex operator algebra sense (cf. [Watts]).

In 4.4 we present more examples by using Th. 4.2. The first example is the coset $H \subset G$ where $H$ is the Cartan subgroup of $G$. This coset is related to Parafermions (cf. [DL]). We then consider a "Maverick" coset model considered in [DJ1] and determine the relevant ring structure.

All the groups considered in this paper are assumed to be connected compact Lie groups unless stated otherwise. As we already noted before, $G$ always denotes a simply connected semisimple compact Lie group of type $A$, i.e., $G=S U\left(N_{1}\right) \times$ $S U\left(N_{2}\right) \times \ldots \times S U\left(N_{n}\right)$.

\section{§2. Coset CFT from Algebraic QFT point of View}

2.1 The irreducible conformal precosheaf and its representations. In this subsection we recall the basic properties enjoyed by the family of the von Neumann algebras associated with a conformal quantum field theory on $S^{1}$ (cf. [GL] and $[\mathrm{FG}])$. By an interval in this paper we shall always mean an open connected subset $I$ of $S^{1}$ such that $I$ and the interior $I^{\prime}$ of its complement are non-empty. We shall denote by $\mathcal{I}$ the set of intervals in $S^{1}$. We shall denote by $P S L(2, \mathbf{R})$ the group of conformal transformations on the complex plane that preserve the orientation and leave the unit circle $S^{1}$ globally invariant. Denote by $\mathbf{G}$ the universal covering group of $P S L(2, \mathbf{R})$. Notice that $\mathbf{G}$ is a simple Lie group and has a natural action on the unit circle $S^{1}$.

Denote by $R(\vartheta)$ the (lifting to $\mathbf{G}$ of the) rotation by an angle $\vartheta$. This oneparameter subgroup of $\mathbf{G}$ will be referred to as rotation group (denoted by Rot) in the following. We may associate a one-parameter group with any interval $I$ in the following way. Let $I_{1}$ be the upper semi-circle, i.e. the interval $\left\{e^{i \vartheta}, \vartheta \in(0, \pi)\right\}$. By using the Cayley transform $C: S^{1} \rightarrow \mathbf{R} \cup\{\infty\}$ given by $z \rightarrow-i(z-1)(z+1)^{-1}$, we may identify $I_{1}$ with the positive real line $\mathbf{R}_{+}$. Then we consider the one-parameter group $\Lambda_{I_{1}}(s)$ of diffeomorphisms of $S^{1}$ such that

$$
C \Lambda_{I_{1}}(s) C^{-1} x=e^{s} x, \quad s, x \in \mathbf{R} .
$$

We also associate with $I_{1}$ the reflection $r_{I_{1}}$ given by

$$
r_{I_{1}} z=\bar{z}
$$

where $\bar{z}$ is the complex conjugate of $z$. It follows from the definition that $\Lambda_{I_{1}}$ restricts to an orientation preserving diffeomorphisms of $I_{1}, r_{I_{1}}$ restricts to an orientation reversing diffeomorphism of $I_{1}$ onto $I_{1}^{\prime}$. 
Then, if $I$ is an interval and we choose $g \in \mathbf{G}$ such that $I=g I_{1}$ we may set

$$
\Lambda_{I}=g \Lambda_{I_{1}} g^{-1}, \quad r_{I}=g r_{I_{1}} g^{-1}
$$

Let $r$ be an orientation reversing isometry of $S^{1}$ with $r^{2}=1$ (e.g. $r_{I_{1}}$ ). The action of $r$ on $P S L(2, \mathbf{R})$ by conjugation lifts to an action $\sigma_{r}$ on $\mathbf{G}$, therefore we may consider the semidirect product of $\mathbf{G} \times{ }_{\sigma_{r}} \mathbf{Z}_{2}$. Since $\mathbf{G} \times{ }_{\sigma_{r}} \mathbf{Z}_{2}$ is a covering of the group generated by $P S L(2, \mathbf{R})$ and $r, \mathbf{G} \times{ }_{\sigma_{r}} \mathbf{Z}_{2}$ acts on $S^{1}$. We call (anti-)unitary a representation $U$ of $\mathbf{G} \times{ }_{\sigma_{r}} \mathbf{Z}_{2}$ by operators on $\mathcal{H}$ such that $U(g)$ is unitary, resp. antiunitary, when $g$ is orientation preserving, resp. orientation reversing.

Now we are ready to define a conformal precosheaf.

An irreducible conformal precosheaf $\mathcal{A}$ of von Neumann algebras on the intervals of $S^{1}$ is a map

$$
I \rightarrow \mathcal{A}(I)
$$

from $\mathcal{I}$ to the von Neumann algebras on a separable Hilbert space $\mathcal{H}$ that verifies the following properties:

A. Isotony. If $I_{1}, I_{2}$ are intervals and $I_{1} \subset I_{2}$, then

$$
\mathcal{A}\left(I_{1}\right) \subset \mathcal{A}\left(I_{2}\right)
$$

B. Conformal invariance. There is a nontrivial unitary representation $U$ of $\mathbf{G}$ on $\mathcal{H}$ such that

$$
U(g) \mathcal{A}(I) U(g)^{*}=\mathcal{A}(g I), \quad g \in \mathbf{G}, \quad I \in \mathcal{I} .
$$

C. Positivity of the energy. The generator of the rotation subgroup $U(R(\vartheta))$ is positive.

D. Locality. If $I_{0}, I$ are disjoint intervals then $\mathcal{A}\left(I_{0}\right)$ and $A(I)$ commute.

The lattice symbol $\vee$ will denote 'the von Neumann algebra generated by'.

E. Existence of the vacuum. There exists a unit vector $\Omega$ (vacuum vector) which is $U(\mathbf{G})$-invariant and cyclic for $\vee_{I \in \mathcal{I}} \mathcal{A}(I)$.

F. Irreducibility. The only $U(\mathbf{G})$-invariant vectors are the scalar multiples of $\Omega$. The term irreducibility is due to the fact (cf. Prop. 1.2 of [GL]) that under the assumption of $\mathbf{F} \vee_{I \in \mathcal{I}} A(I)=B(\mathcal{H})$.

We have the following (cf. Prop. 1.1 of $[\mathrm{GL}]$ ):

2.1 Proposition. Let $\mathcal{A}$ be an irreducible conformal precosheaf. The following hold:

(a) Reeh-Schlieder theorem: $\Omega$ is cyclic and separating for each von Neumann algebra $\mathcal{A}(I), I \in \mathcal{I}$. 
(b) Bisognano-Wichmann property: $U$ extends to an (anti-)unitary representation of $\mathbf{G} \times{ }_{\sigma_{r}} \mathbf{Z}_{2}$ such that, for any $I \in \mathcal{I}$,

$$
\begin{aligned}
U\left(\Lambda_{I}(2 \pi t)\right) & =\Delta_{I}^{i t} \\
U\left(r_{I}\right) & =J_{I}
\end{aligned}
$$

where $\Delta_{I}, J_{I}$ are the modular operator and the modular conjugation associated with $(\mathcal{A}(I), \Omega)$. For each $g \in \mathbf{G} \times_{\sigma_{r}} \mathbf{Z}_{2}$

$$
U(g) \mathcal{A}(I) U(g)^{*}=\mathcal{A}(g I) .
$$

(c) Additivity: if a family of intervals $I_{i}$ covers the interval $I$, then

$$
\mathcal{A}(I) \subset \vee_{i} \mathcal{A}\left(I_{i}\right) .
$$

(d) Haag duality: $\mathcal{A}(I)^{\prime}=\mathcal{A}\left(I^{\prime}\right)$.

Assume $\mathcal{A}$ is an irreducible conformal precosheaf as defined in above. By Cor. B.2 of [GL], the only invariant vectors under the action of the one-parameter group $U\left(\Lambda_{I}(2 \pi t)\right)$ are the scalar multiples of $\Omega$. This fact and (b) of Prop. 2.1 are usually referred to as the action of modular group is ergodic and geometric respectively. It follows that $\mathcal{A}(I)$ is a type $I I I_{1}$ factor for any $I \in \mathcal{I}$ (cf. Prop. 1.2 of [GL]).

A covariant representation $\pi$ of $\mathcal{A}$ is a family of representations $\pi_{I}$ of the von Neumann algebras $\mathcal{A}(I), I \in \mathcal{I}$, on a separable Hilbert space $\mathcal{H}_{\pi}$ and a unitary representation $U_{\pi}$ of the covering group $\mathbf{G}$ of $\operatorname{PSL}(2, \mathbf{R})$ such that the following properties hold:

$$
\begin{gathered}
\left.I \subset \bar{I} \Rightarrow \pi_{\bar{I}}\right|_{\mathcal{A}(I)}=\pi_{I} \quad \text { (isotony) } \\
\operatorname{ad} U_{\pi}(g) \cdot \pi_{I}=\pi_{g I} \cdot \operatorname{ad} U(g) \text { (covariance) } .
\end{gathered}
$$

A covariant representation $\pi$ is called irreducible if $\vee_{I \in \mathcal{I}} \pi(\mathcal{A}(I))=B\left(\mathcal{H}_{\pi}\right)$. By our definition the irreducible conformal precosheaf is in fact an irreducible representation of itself and we will call this representation the vacuum representation. Note that by Cor. B.2 of [GL], the vacuum representation is the unique (up to unitary equivalence) irreducible covariant representation which contains an eigenvector of the generator of the rotation group with lowest eigenvalue 0 . A unitary equivalence class of covariant representations of $\mathcal{A}$ is called a superselection sector (cf. Page 17 of $[\mathrm{GL}])$.

The composition of two superselection sectors, similar to the Connes's fusion (cf . Chap. V of [W2]), can be defined (cf. $\S I V .2$ of [FG] ). The composition is manifestly unitary, and this is one of the virtues of the above formulation.

Now let $G$ the group as in the introduction . Let $g=\operatorname{Lie}(G), g_{\mathbb{C}}:=g \otimes \mathbb{C}$. Denote by $\hat{g}$ the affine Kac-Moody algebra (cf. P. 163 of $[\mathrm{KW}]$ ) associated to $g_{\mathbb{C}}$. Recall $\hat{g}=g_{\mathbb{C}} \otimes \mathbb{C}\left[t, t^{-1}\right] \oplus \mathbb{C} c$, where $\mathbb{C} c$ is the 1-dimensional center of $\hat{g}$. 
Denote by $L G$ the group of smooth maps $f: S^{1} \mapsto G$ under pointwise multiplication. The diffeomorphism group of the circle Diff $S^{1}$ is naturally a subgroup of $\operatorname{Aut}(L G)$ with the action given by reparametrization. In particular $\mathbf{G}$ acts on $L G$. We will be interested in the projective unitary representations (cf. Chap. 9 of $[\mathrm{PS}]) \pi$ of $L G$ that are both irreducible and have positive energy. This implies that $\pi$ should extend to $L G \ltimes$ Rot so that the generator of the rotation group Rot is positive. It follows from Chap. 9 of [PS] or P. 490 of [W2] that for fixed level (a finite set of positive integers, see the footnote on this page), there are only finite number of such irreducible projective representations (cf. 4.3 for a list in the case $G=S U(N)$ ). These irreducible projective representations can be obtained by exponentiating the integrable representations of $\hat{g}$ at the same level by Th. 4.4 of $[\mathrm{GW}]$. We refer the reader to III.7 of [FG] for a summary about the properties of these representations.

Now let $H \subset G$ be a connected Lie subgroup. Let $\pi^{i}$ be an irreducible projective representations of $L G$ with positive energy at level $L^{1}$ on Hilbert space $H^{i}$. When restricting to $L H, \pi^{i}$ is a projective representation of $L H$ with positive energy. By the proposition on P. 484 of [W2], $\pi^{i}$ is a direct sum of irreducible projective representations of $L H$. Suppose when restricting to $L H, H^{i}$ decomposes as:

$$
H^{i}=\sum_{\alpha} H_{i, \alpha} \otimes H_{\alpha}
$$

and $\pi_{\alpha}$ are irreducible projective representations of $L H$ on Hilbert space $H_{\alpha}$. The set of $(i, \alpha)$ which appears in the above decompositions will be denoted by exp. The above decomposition can also be obtained via exponentiation (cf. Th. 4.4 of [GW]) from a similar decomposition (cf. (1.6.2) of $[\mathrm{KW}]$ ) of integrable representation of $\hat{g}$ when restricting to $\hat{h}$ (the affine Kac-Moody algebra associated with $H$ ).

We shall use $\pi^{0}$ (resp. $\pi_{0}$ ) to denote the vacuum representation of $L G$ (resp. $L H$ ) on $H^{0}$ (resp. $H_{0}$ ). This is the unique projective representation of $L G$ (resp. $L H)$ which contains a nonzero vector, unique up to multiplication by a nonzero scalar, with the property that it is an eigenvector of the generator of rotation group with eigenvalue 0 . Such vectors will be called vacuum vectors. By Th. 3.2 of $[\mathrm{FG}]$ (also cf. $\S 17$ of [W2]) the vacuume representation $\pi^{0}$ of $L G$ gives rise to to an irreducible conformal precosheaf by the map $I \in \mathcal{I} \rightarrow \pi^{0}\left(L_{I} G\right)^{\prime \prime}$ on $H^{0}$. Similarly $I \in \mathcal{I} \rightarrow \pi_{0}\left(L_{I} H\right)^{\prime \prime}$ on $H_{0}$ is an irreducible conformal precosheaf on $H_{0}$.

Let $\Omega\left(\right.$ resp. $\left.\Omega_{0}\right)$ be a vacuum vector in $\pi^{0}$ (resp. $\left.\pi_{0}\right)$ and assume

$$
\Omega=\Omega_{0,0} \otimes \Omega_{0}
$$

with $\Omega_{0,0} \in H_{0,0}$. We shall always assume that $H \subset G_{L}$ is not a conformal inclusion since the case of conformal inclusions has been considered in [X1]. For the definition of conformal inclusion, we refer the reader to P. 210 of $[\mathrm{KW}]$.

\footnotetext{
${ }^{1}$ When $\mathrm{G}$ is the direct product of simple groups, $L$ is a multi-index, i.e., $L=\left(L_{1}, \ldots, L_{n}\right)$, where $L_{i} \in \mathbb{N}$ corresponding to the level of the $i$-th simple group. To save some writing we write the coset as $H \subset G_{L}$ or as $H \subset G$ when the level is kept fixed in the question.
} 
Lemma 2.1. (1) $\pi^{0}\left(L_{I} H\right)^{\prime \prime}$ is strongly additive, i.e., if $I_{1}, I_{2}$ are the connected components of the interval I with one internal point removed, then:

$$
\pi^{0}\left(L_{I} H\right)^{\prime \prime}=\pi^{0}\left(L_{I_{1}} H\right)^{\prime \prime} \vee \pi^{0}\left(L_{I_{2}} H\right)^{\prime \prime}
$$

(2) $\pi^{0}\left(L_{I} H\right)^{\prime} \cap \pi^{0}\left(L_{I^{\prime}} H\right)^{\prime}=\pi^{0}(L H)^{\prime}$.

Proof. Ad (1): Let $P_{0}$ be the projection from $H^{0}$ onto $\Omega_{0,0} \otimes H_{0}$. Since the action of the modular group of $\pi^{0}\left(L_{J} G\right)^{\prime \prime}$ with respect to $\Omega$ is geometric and ergodic, it fixes globally $\pi^{0}\left(L_{J} H\right)^{\prime \prime}$, and by Takesaki's theorem (cf. [MT] or P. 495 of [W2]), $\pi^{0}\left(L_{J} H\right)^{\prime \prime}$ is a factor. So the map

$$
x \in \pi^{0}\left(L_{J} H\right)^{\prime \prime} \rightarrow x P_{0}
$$

is a $*$-isomorphism (cf. P. 492 of [W2]). Hence to prove (1) we just need to show

$$
\pi_{0}\left(L_{I} H\right)^{\prime \prime}=\pi_{0}\left(L_{I_{1}} H\right)^{\prime \prime} \vee \pi_{0}\left(L_{I_{2}} H\right)^{\prime \prime} .
$$

By Haag duality in Prop. 2.1 it is enough to show

$$
\pi_{0}\left(L_{I^{\prime}} H\right)^{\prime \prime}=\pi_{0}\left(L_{I_{1}^{\prime}} H\right)^{\prime \prime} \cap \pi_{0}\left(L_{I_{2}^{\prime}} H\right)^{\prime \prime} .
$$

By Reeh-Schlider theorem in Prop. 2.1, the closed space spanned by $\pi^{0}\left(L_{J} H\right) \Omega$ is $P_{0} H^{0}$ for any interval $J$, and by Takesaki's theorem (cf. [MT] or (c) of Theorem on P. 495 of [W2]),

$$
\pi^{0}\left(L_{J} H\right)^{\prime \prime}=\left\{\mathbb{C} P_{0}\right\}^{\prime} \cap \pi^{0}\left(L_{J} G\right)^{\prime \prime},
$$

so to prove (1) we just have to show that

$$
\pi^{0}\left(L_{I^{\prime}} G\right)^{\prime \prime}=\pi^{0}\left(L_{I_{1}^{\prime}} G\right)^{\prime \prime} \cap \pi^{0}\left(L_{I_{2}^{\prime}} G\right)^{\prime \prime}
$$

It is sufficient to show the above in the case when $G$ is simple, and this follows from Th. E of [W2].

Ad (2): Let $I_{1}=I, I_{2}, I_{3}, I_{4}$ be four consecutive disjoint intervals on $S^{1}$ such that the closure of $I \cup I_{2} \cup I_{3} \cup I_{4}$ is $S^{1}$. Let $J_{1}=I_{1}^{\prime}, J_{3}=I_{3}^{\prime}$. For any $a \in L H$, since $H$ is connected, we can always choose $a_{1} \in L_{J_{1}} H$ such that $a_{1}\left|\tilde{I}_{1}=e, a_{1}\right| \tilde{I}_{3}=a \mid \tilde{I}_{3}$, where $e$ is the identity of $G, I_{1} \subset \tilde{I}_{1}, I_{3} \subset \tilde{I}_{3}$, and the closure of $\tilde{I}_{1}$ and the closure of $\tilde{I}_{3}$ are disjoint. Let $a_{2}=a a_{1}^{-1}$, then $a_{2} \in L_{J_{3}} H$, and $a_{2} a_{1}=a$. Hence $L_{J_{1}} H$ and $L_{J_{3}} H$ generate $L H$ algebraically, and

$$
\pi^{0}\left(L_{J_{1}} H\right)^{\prime \prime} \vee \pi^{0}\left(L_{J_{3}} H\right)^{\prime \prime}=\pi^{0}(L H)^{\prime \prime} .
$$

By (1)

$$
\begin{aligned}
& \pi^{0}\left(L_{I_{2}} H\right)^{\prime \prime} \vee \pi^{0}\left(L_{I_{3}} H\right)^{\prime \prime} \vee \pi^{0}\left(L_{I_{4}} H\right)^{\prime \prime}=\pi^{0}\left(L_{J_{1}} H\right)^{\prime \prime} ; \\
& \pi^{0}\left(L_{I_{1}} H\right)^{\prime \prime} \vee \pi^{0}\left(L_{I_{2}} H\right)^{\prime \prime} \vee \pi^{0}\left(L_{I_{4}} H\right)^{\prime \prime}=\pi^{0}\left(L_{J_{3}} H\right)^{\prime \prime} ;
\end{aligned}
$$


Hence

$$
\pi^{0}\left(L_{J_{1}} H\right)^{\prime \prime} \vee \pi^{0}\left(L_{J_{3}} H\right)^{\prime \prime} \subset \pi^{0}\left(L_{I} H\right)^{\prime \prime} \vee \pi^{0}\left(L_{I^{\prime}} H\right)^{\prime \prime},
$$

and

$$
\pi^{0}(L H)^{\prime \prime}=\pi^{0}\left(L_{I} H\right)^{\prime \prime} \vee \pi^{0}\left(L_{I^{\prime}} H\right)^{\prime \prime} .
$$

By taking the commutant of the above equality, the proof of (2) is complete.

For each interval $I \subset S^{1}$, we define:

$$
A(I):=\pi^{0}\left(L_{I} H\right)^{\prime} \cap \pi^{0}\left(L_{I} G\right)^{\prime \prime} P,
$$

where $P$ is the projection from $H^{0}$ to closed subspace $\mathcal{H}$ spanned by

$\vee_{J \in \mathcal{I}} \pi^{0}\left(L_{J} H\right)^{\prime} \cap \pi^{0}\left(L_{J} G\right) \Omega$. Note that

$$
\pi^{0}\left(L_{I} H\right)^{\prime} \cap \pi^{0}\left(L_{I} G\right)^{\prime \prime} \supset \pi^{0}(L H)^{\prime} \cap \pi^{0}\left(L_{I} G\right)^{\prime \prime} .
$$

On the other hand if $x \in \pi^{0}\left(L_{I} H\right)^{\prime} \cap \pi^{0}\left(L_{I} G\right)^{\prime \prime}$, then $x \in \pi^{0}\left(L_{I} H\right)^{\prime} \cap \pi^{0}\left(L_{I^{\prime}} H\right)^{\prime}$, but $\pi^{0}\left(L_{I} H\right)^{\prime} \cap \pi^{0}\left(L_{I^{\prime}} H\right)^{\prime}=\pi^{0}(L H)^{\prime}$ by (2) of lemma 2.1, so $x \in \pi^{0}(L H)^{\prime}$. This shows that

$$
\pi^{0}\left(L_{I} H\right)^{\prime} \cap \pi^{0}\left(L_{I} G\right)^{\prime \prime}=\pi^{0}(L H)^{\prime} \cap \pi^{0}\left(L_{I} G\right)^{\prime \prime} .
$$

It follows that if $x \in \pi^{0}\left(L_{I} H\right)^{\prime} \cap \pi^{0}\left(L_{I} G\right)^{\prime \prime}$, then as an operator on $H^{0}$, it takes the form $\oplus_{0, \alpha} B\left(H_{0, \alpha}\right) \otimes i d_{\alpha}$, and $x \Omega \in H_{0,0} \otimes \Omega_{0}$, so we have $\mathcal{H} \subset H_{0,0} \otimes \Omega_{0}$.

Proposition 2.2. The map $I \in \mathcal{I} \rightarrow A(I)$ as defined above is an irreducible conformal precosheaf.

Proof. We have to check conditions $\mathbf{A}$ to $\mathbf{F}$. A follows from

$$
\pi^{0}\left(L_{I} H\right)^{\prime} \cap \pi^{0}\left(L_{I} G\right)^{\prime \prime}=\pi^{0}(L H)^{\prime} \cap \pi^{0}\left(L_{I} G\right)^{\prime \prime}
$$

which is proved above, $\mathbf{B}$ and $\mathbf{C}$ follows from a lemma on P. 485 of [W2] except that we need to show that the action of $\mathbf{G}$ is nontrivial. Denote by $U(t)$ the action of the rotation group on $H_{0,0}$ and assume $U(t)=\exp \left(2 \pi i L_{0}^{g, h} t\right)$, where $L_{0}^{g, h}$ is the positive self-adjoint operator. Fix $\tau \in \mathbb{C}$ with $\operatorname{Im} \tau>0$. Then

$$
b_{0}^{0}(\tau):=\exp \left((1 / 24) 2 \pi i \tau\left(z_{m}-\dot{z}_{\dot{m}}\right)\right) t r_{H_{0,0}} \exp \left(2 \pi i \tau L_{0}^{g, h}\right)
$$

is a branching function (cf. 3.2 .7 of $[\mathrm{KW}]$ ), where $\left(z_{m}-\dot{z}_{\dot{m}}\right)$ is a nonnegative number defined as 1.4.2 of [KW]. We will not need the detailed expression of the branching function in our argument, all we need to know is if $H \subset G$ is not conformal, then $z_{m}-\dot{z}_{\dot{m}}>0$ (cf. P. 210 of $[\mathrm{KW}]$ ). Let us show that if $H \subset G$ is not conformal which is assumed throughout this paper, then $L_{0}^{g, h} \neq 0$. If $L_{0}^{g, h}=0$, then $b_{0}^{0}(\tau)=$ $\exp \left((1 / 24) 2 \pi i \tau\left(z_{m}-\dot{z}_{\dot{m}}\right)\right) \operatorname{dim}\left(H_{0,0}\right)$. Since $b_{0}^{0}(\tau)$ is well defined for $\operatorname{Im} \tau>0$ (cf. 
P. 170 of $[\mathrm{KW}]$ ), we must have $\operatorname{dim}\left(H_{0,0}\right)<\infty$, but by ${ }^{2}$ (a) of Th. B of $[\mathrm{KW}]$, $b_{0}^{0}(\tau) \sim b(0,0) \exp \left(\frac{\pi i\left(z_{m}-\dot{z}_{\dot{m}}\right)}{12 \tau}\right)$ as $\tau \rightarrow 0$ where $b(0,0)>0$. This is a contradiction, and shows that $L_{0}^{g, h} \neq 0$. So the action of $\mathbf{G}$ is nontrivial. $\mathbf{F}$ follows from the uniqueness of the vacuum $\Omega$ (up to multiplication by a non-zero scalar) for $L G$. D and $\mathbf{E}$ follow from the definitions.

The irreducible conformal precosheaf as in Prop. 2.2 is defined to be the irreducible conformal precosheaf of the coset $H \subset G_{L}$. Note that when $H=\{e\}$ is a trivial subgroup ( $e$ denotes the identity element in $G$ ), the irreducible conformal precosheaf defined above coincides with the one defined in III.8 of [FG]. Since the action of the modular group of $\pi^{0}\left(L_{I} G\right)^{\prime \prime}$ with respect to $\Omega$ is geometric and ergodic, it fixes globally $\pi^{0}\left(L_{I} H\right)^{\prime \prime}$, hence $\pi^{0}\left(L_{I} G\right)^{\prime \prime} \cap \pi^{0}\left(L_{I} H\right)^{\prime}$, and by Takesaki's theorem (cf. [MT] or P. 495 of [W2]), $\pi^{0}\left(L_{I} G\right)^{\prime \prime} \cap \pi^{0}\left(L_{I} H\right)^{\prime}$ is a factor. It follows that the map

$$
x \in \pi^{0}\left(L_{I} G\right)^{\prime \prime} \cap \pi^{0}\left(L_{I} H\right)^{\prime} \rightarrow x P \in \pi^{0}\left(L_{I} G\right)^{\prime \prime} \cap \pi^{0}\left(L_{I} H\right)^{\prime} P
$$

is a $*$ isomorphism (cf. P. 492 of [W2]), and can be implemented by a unitary $U_{1}: H^{0} \rightarrow \mathcal{H}$, i.e., $x=U_{1}^{*} x P U_{1}$, since $\pi^{0}\left(L_{I} G\right)^{\prime \prime} \cap \pi^{0}\left(L_{I} H\right)^{\prime} P$ is a type $I I I_{1}$ factor by Prop. 2.2 and the remarks after Prop. 2.1.

Let us define a class of covariant representations of $A(I)$ coming from the decompositions of irreducible projective representations $\pi^{i}$ of $L G$ with respect to $L H$. By the remarks after Th. B on P. 502 of [W2], for any fixed interval $I$, there exists a unitary map $U: H^{i} \rightarrow H^{0}$ such that

$$
\pi^{i}(a)=U^{*} \pi^{0}(a) U, \forall a \in L_{I} G .
$$

For $y=x P \in \pi^{0}\left(L_{I} H\right)^{\prime} \cap \pi^{0}\left(L_{I} G\right)^{\prime} P$, we define

$$
\pi^{i}(y)=U^{*} U_{1}^{*} y U_{1} U .
$$

This gives a factor representation of $A(I)$. Let $P_{i, \alpha}$ be a projection from $H^{i}$ to a subspace $H_{i, \alpha} \otimes \Omega_{\alpha}$ where $\Omega_{\alpha}$ is a unit vector in $H_{\alpha}$. Then

$$
y \in A(I) \rightarrow \pi_{i, \alpha}(y):=\pi^{i}(y) P_{i, \alpha}
$$

is a subrepresentation of the factor representation $\pi^{i}$, and so the map above is a *-isomorphism (cf. P. 492 of [W2]). Denote by $\pi^{i}(g)$ the action of $g \in \mathbf{G}$ on $H^{i}$. By the lemma on P. 485 of [W2], $\pi^{i}(g)$ can be written as $\pi^{i}(g)=\oplus_{\alpha} \pi_{i, \alpha}(g) \otimes \pi_{\alpha}(g)$. One checks by using the definitions that the representations $\pi_{i, \alpha}$ of $A(I)$ and the

\footnotetext{
${ }^{2}$ The branching function here corresponds to $b_{0}^{0}$ in the notations of $[\mathrm{KW}]$ on P. 187 , where 0 always denotes the vacuum representations. The assumption of Th. B of $[\mathrm{KW}]$ follows from the definition 2.5.4 of $[\mathrm{KW}]$ when $\Lambda=0, \lambda=0$.
} 
representations $\pi_{i, \alpha}$ of $\mathbf{G}$ satisfy the covariance condition, and so $\pi_{i, \alpha}$ are covariant representations of $A(I)$. The study of these representations is the main purpose of this paper.

By the same argument as in the proof of (1) of lemma 2.1 one can show that $A(I)$ as in Prop. 2.2 is strongly additive, i.e., if $I_{1}, I_{2}$ are the connected components of the interval $I$ with one internal point removed, then:

$$
A(I)=A\left(I_{1}\right) \vee A\left(I_{2}\right)
$$

In fact, by Haag duality in Prop. 2.1, it is sufficient to show that

$$
A\left(I^{\prime}\right)=A\left(I_{1}^{\prime}\right) \cap A\left(I_{2}^{\prime}\right)
$$

which is equivalent to

$$
\pi^{0}\left(L_{I^{\prime}} H\right)^{\prime} \cap \pi^{0}\left(L_{I^{\prime}} G\right)^{\prime \prime}=\left(\pi^{0}\left(L_{I_{1}^{\prime}} H\right)^{\prime} \cap \pi^{0}\left(L_{I_{1}^{\prime}} G\right)^{\prime \prime}\right) \cap\left(\pi^{0}\left(L_{I_{2}^{\prime}} H\right)^{\prime} \cap \pi^{0}\left(L_{I_{2}^{\prime}} G\right)^{\prime \prime}\right)
$$

by the paragraph after Prop. 2.2. Let $P$ be the projection defined before Prop. 2.2. By Reeh-Schlider theorem in Prop. 2.1 the closed space spanned by $\pi^{0}\left(L_{I^{\prime}} H\right)^{\prime} \cap$ $\pi^{0}\left(L_{I^{\prime}} G\right)^{\prime \prime} \Omega$ is $P H^{0}$ for any interval $I$, and by Takesaki's theorem (cf. [MT] or (c) of Theorem on P. 495 of [W2]),

$$
\pi^{0}\left(L_{I^{\prime}} H\right)^{\prime} \cap \pi^{0}\left(L_{I^{\prime}} G\right)^{\prime \prime}=\{\mathbb{C} P\}^{\prime} \cap \pi^{0}\left(L_{I^{\prime}} G\right)^{\prime \prime},
$$

so we just have to show that

$$
\pi^{0}\left(L_{I^{\prime}} G\right)^{\prime \prime}=\pi^{0}\left(L_{I_{1}^{\prime}} G\right)^{\prime \prime} \cap \pi^{0}\left(L_{I_{2}^{\prime}} G\right)^{\prime \prime}
$$

It is sufficient to show the above equation in the case $G$ is simple, and in this case it follows from Th. E of [W2].

Note that the inclusion

$$
\left(\pi^{0}\left(L_{I} G\right)^{\prime \prime} \cap \pi^{0}\left(L_{I} H\right)^{\prime}\right) \vee \pi^{0}\left(L_{I} H\right)^{\prime \prime} \subset \pi^{0}\left(L_{I} G\right)^{\prime \prime}
$$

is irreducible. In fact

$$
\begin{aligned}
& \left(\left(\pi^{0}\left(L_{I} G\right)^{\prime \prime} \cap \pi^{0}\left(L_{I} H\right)^{\prime}\right) \vee \pi^{0}\left(L_{I} H\right)^{\prime \prime}\right)^{\prime} \cap \pi^{0}\left(L_{I} G\right)^{\prime \prime} \\
& =\left(\pi^{0}\left(L_{I} G\right)^{\prime \prime} \cap \pi^{0}\left(L_{I} H\right)^{\prime}\right)^{\prime} \cap\left(\pi^{0}\left(L_{I} G\right)^{\prime \prime} \cap \pi^{0}\left(L_{I} H\right)^{\prime}\right)=\mathbb{C}
\end{aligned}
$$

since $\pi^{0}\left(L_{I} G\right)^{\prime \prime} \cap \pi^{0}\left(L_{I} H\right)^{\prime}$ is a factor by the paragraph after Prop. 2.2. This fact can also be proved by using the fact that in $H_{0,0}$, the vacuum representation of $A(I)$ appears once and only once. We shall see in the next subsection that $\pi_{0,0}$ is in fact the vacuum representation under general conditions. 
$2.2 \pi_{0,0}$ is the vacuum Representation. As a first step in the study of representations $\pi_{i, \alpha}$ we show that the representation of $\pi_{0,0}$ on $H_{0,0}$ is the vacuum representation. This is equivalent to $\mathcal{H}=H_{0,0} \otimes \Omega_{0}$ by definition.

Theorem 2.3. Suppose $H \subset G$, and $H$ is simply connected. Then $\mathcal{H}=H_{0,0} \otimes \Omega_{0}$. Hence $\pi_{0,0}$ is the vacuum representation.

The idea of the proof is to use smeared vertex operators. From the proof one can also see the close relation between our irreducible conformal precosheaf and the definition of coset vertex operator algebra in $\S 5$ of $[\mathrm{FZ}]$. In fact, the coset vertex operator algebra in $\S 5$ of [FZ] can be thought as "germs" of ours.

Let $g($ resp. $h)$ be the Lie algebra of $G$ (resp. $H)$. Choose a basis $e_{\alpha}, e_{-\alpha}, h_{\alpha}$ in $g_{\mathbb{C}}:=g \otimes \mathbb{C}$ with $\alpha$ ranging over the set of roots as in $\S 2.5$ of [PS]. Let $X_{\alpha}:=$ $e_{\alpha}+e_{-\alpha}, Y_{\alpha}:=i\left(e_{\alpha}-e_{-\alpha}\right)$. Denote by $\hat{g}$ the affine Kac-Moody algebra (cf. P. 163 of $[\mathrm{KW}])$ associated to $g_{\mathbb{C}}$. Note $\hat{g}=g_{\mathbb{C}} \otimes \mathbb{C}\left[t, t^{-1}\right] \oplus \mathbb{C} c$, where $\mathbb{C} c$ is the 1-dimensional center of $\hat{g}$. For $X \in g$, Define $X(n):=X \otimes t^{n}, X(z):=\sum_{n} X(n) z^{-n-1}$ as on Page 312 of $[\mathrm{KT}]$ and $X^{+}(z):=\sum_{n<0} X(n) z^{-n-1}, X^{-}(z):=\sum_{n \geq 0} X(n) z^{-n-1}$.

Let $\pi^{0}$ be the vacuum representation of $L G$ on $H^{0}$ with vacuum vector $\Omega$. Let $D$ be the generator of the action of the rotation group on $H^{0}$. For $\xi \in H^{0}$, we define $\|x\|_{s}=\left\|(1+D)^{s} x\right\|, s \in \mathbb{R} . H^{0, s}:=\left\{x \in H^{0} \mid\|x\|_{s}<\infty\right\}$ and $H_{\infty}^{0}=\cap_{s \in \mathbb{R}} H^{0, s}$. Note that when $s \geq 0, H^{0, s}$ is a complete space under the norm $\|\cdot\|_{s}$. $H_{0}^{0}$ will denote the finite linear sum of the eigenvectors of $D$. Clearly $H_{0}^{0} \subset H_{\infty}^{0}$. The elements of $H_{0}^{0}$ (resp. $H_{\infty}^{0}$ ) will be called finite energy vectors (resp. smooth vectors). The eigenvalue of $D$ is sometimes referred to as energy.

Let us recall a few elementary facts about vertex operators which will be used. See $[\mathrm{FLM}],[\mathrm{Kacv}]$ or $[\mathrm{D}]$ for an introduction on vertex operator algebras. Define $\operatorname{End}\left(H_{0}^{0}\right)$ to be the space of all linear operators (not necessarily bounded) from $H_{0}^{0}$ to $H_{0}^{0}$ and set

$$
\operatorname{End}\left(H_{0}^{0}\right)\left[\left[z, z^{-1}\right]\right]:=\left\{\sum_{n \in \mathbb{Z}} v_{n} z^{n} \mid v_{n} \in \operatorname{End}\left(H_{0}^{0}\right)\right\}
$$

By the statement on P. 154 of [FZ] which follows from Th. 2.4.1 of [FZ] there exists a linear map

$$
\xi \in H_{0}^{0} \rightarrow V(\xi, z)=\sum_{m \in \mathbb{Z}} \xi(m) z^{-m-1} \in \operatorname{End}\left(H_{0}^{0}\right)\left[\left[z, z^{-1}\right]\right]
$$

with the following properties:

(1) $\xi(-1) \Omega=\xi$;

(2) If

$$
\xi=X_{i_{1}}(-1) \ldots X_{i_{t}}(-1) \Omega
$$

then

$$
V(\xi, z)=: X_{i_{1}}(z) \ldots X_{i_{t}}(z):
$$


where :, : are normal ordered products (cf. (2.38), (2.39) of [D]).

$V(\xi, z)$ is called a vertex operator of $\xi$.

Let $\psi \in H_{0,0} \otimes \Omega_{0}$ be an eigenvector of $D$ with eigenvalue $n \in \mathbb{N}$. Then $\psi$ takes the form:

$$
\psi=\sum C_{i_{1}, \ldots, i_{t}} X_{i_{1}}\left(-n_{1}\right) \ldots X_{i_{t}}\left(-n_{t}\right) \Omega
$$

where the sum is finite,$n_{i} \geq 0$ and $C_{i_{1}, \ldots, i_{t}} \in \mathbb{C}$. Since $g$ is semi-simple, $g=[g, g]$, so any $X(-n), n>1$ can be expressed in terms of linear combinations of the form

$$
X(-1) Y(-1) \ldots Z(-1) \text {. }
$$

Because $\Omega$ is the vacuum, $X \Omega=0, \forall X \in g$, so in

$$
X_{i_{1}}\left(-n_{1}\right) \ldots X_{i_{t}}\left(-n_{t}\right) \Omega
$$

if certain $X \in g$ appears, we can always move $X$ to the right until it vanishes when acting on $\Omega$. For the above two reasons, we can assume that $n_{1}=\ldots=n_{t}=1$. The vertex operator $V(\psi, z)$ is then given by:

$$
V(\psi, z)=\sum C_{i_{1}, \ldots, i_{t}}: X_{i_{1}}(z) \ldots X_{i_{t}}(z):
$$

where :, : are normal ordered products by property (2) above.

Recall $V(\psi, z)=\sum_{m} \psi(m) z^{-m-1}$. Define

$$
V(m):=\psi(m+n-1)
$$

so we have $V(\psi, z)=\sum_{m} V(m) z^{-m-n}$. This expression for $V(\psi, z)$ is in accordance with the convention of $[\mathrm{KT}]$. Note that $V(-n) \Omega=\psi(-1) \Omega=\psi$ by property $(1)$ above.

Let $f=\sum_{m} f(m) z^{m}$ be a test function with only a finite number of non-zero $f_{m}$. Such $f$ will be referred to as finite energy functions. Define

$$
\|f\|_{s}=\sum_{n \in \mathbb{Z}}(1+|m|)^{s}|f(m)| .
$$

The smeared vertex operator $V(\psi, f)$ is defined to be:

$$
V(\psi, f)=\frac{1}{2 \pi i} \int_{S^{1}} V(\psi, z) f d z=\sum_{m} f(m+n-1) V(m) .
$$

$V(\psi, f)$ is a well defined operator on $H_{0}^{0}$. Let $V(\psi, f)^{F A}$ be the formal adjoint of $V(\psi, f)$ on $H_{0}^{0}$. It is defined by the equation

$$
\langle V(\psi, f) x, y\rangle=\left\langle x, V(\psi, f)^{F A} y\right\rangle, \forall x, y \in H_{0}^{0}
$$

where $\langle$,$\rangle is the inner product on Hilbert space H^{0}$. When no confusion arises, we will write $V(\psi, f)$ simply as $V(f)$. Similarly for $X \in g$, we define $X(f):=$ $\sum_{n} X(n) f(n)$.

When the level is $1 H^{0}$ admits a fermionic representation (cf. $\S 13.3$ of [PS] or I.6 of [W2]), and we will denote the underlying Hilbert space by $F$. In fact, to each simple component $G_{i}$ of $G$ there is a level 1 vacuum fermionic representation of $L G_{i}$ on $F_{i}, i=1, \ldots, m$, and $F=F_{1} \otimes F_{2} \ldots \otimes F_{m}$. 
Lemma 1. (1). Let $\xi \in H_{0}^{0}$, and $f$ is a finite energy function. There exists positive integer $a$ and $c>0$ which are independent of $f$ and $\xi$ such that

$$
\|V(\psi, f) \xi\|_{s} \leq c\|f\|_{|s|+a}\|\xi\|_{s+a}
$$

(2). (1) is also true for $V(\psi, f)^{F A}$ for the same constants $c$ and $a$.

Proof. Note by definition

$$
V(\psi, f)=\frac{1}{2 \pi i} \int_{S^{1}} V(\psi, z) f d z=\sum_{m} f(m+n-1) V(m) .
$$

If we can show (1) for the case $V(\psi, f)=V(l), \forall l$, then

$$
\begin{aligned}
\|V(\psi, f) \xi\|_{s} & =\left\|\sum_{l \in \mathbb{Z}} f(l+n-1) V(l) \xi\right\|_{s} \\
& \leq c \sum_{l \in \mathbb{Z}}(1+|l|)^{|s|+a}|f(l+n-1)|\|\xi\|_{s+a} \\
& \leq c(1+|n|)^{|s|+a}|| f\left\|_{s+a}|| \xi\right\|_{s+a} .
\end{aligned}
$$

So we just need to show (1) for the case $V(\psi, f)=V(l), \forall l$. Also note that $V(\psi, f)$ is linear in $\psi$, so it is sufficient to prove (1) in the case when

$$
V(\psi, z)=: X_{1}(z) \ldots X_{n}(z):
$$

and $\xi$ is an eigenvector of $D$ with eigenvalue $\mu$. Similarly to prove (2) we just need to prove (2) in the case $V(\psi, f)^{F A}=V(l)^{F A}$, and $\psi, \xi$ are as above. Note that

$$
: X_{1}(z) \ldots X_{n}(z):
$$

is a summation of $2^{n}$ of expressions of the form $X_{i}^{+} \ldots X_{j}^{+} X_{i^{\prime}}^{-} \ldots X_{j^{\prime}}^{-}$. We will in fact prove the ineqaulity in lemma 1 for such expressions. This will finish the proof of lemma 1 by definitions. We first prove this for level 1 and $G$ is simple, and the representation is on $F$.

To avoid too many subscripts we will denote the fermionic creation or annilation operators simply by $a(m)$, since we only need to use the fact that $a(m)$ increases the energy by $m$, i.e., $[D, a(m)]=m a(m)$ on $H_{0}^{0}$ and its norm is 1 in our proof. Note in terms of $a(m)$,

$$
X(k)=\sum_{m>0} a(m-k) a(-m)-\sum_{m \geq 0} a(m) a(-m-k)
$$

when acting on finite energy vectors, cf. the expression in a theorem on Page 486 of [W2]. Notice that we have dropped all the subscripts here for simplicity. We will prove the lemma for $V(z)=X^{+}(z) \ldots X^{+}(z) Y^{-}(z) \ldots Y^{-}(z)$ where there are $n$ 
$X, Y$ and we have dropped the subscripts for simplicity. Then $V(l) \xi$ is a sum of $2^{n}$ expressions of the form:

$$
\sum a\left(m_{1}\right) \ldots a\left(m_{2 n}\right) \xi
$$

where the sum is over finite number of $m_{1}, \ldots, m_{n}$ 's, subject to certain constraint. Let us now show by induction on $n$ that

$$
0 \leq\left|m_{i}\right| \leq e(1+|l|+\mu), i=1, \ldots, 2 n,
$$

where $e \geq 1$ depends only on $V(z)$. When $n=1$, it is contained in a proof on page 488 of [W2]. Assume that the statement is true for $k<n$. Suppose $V(z)=Y(z) X^{-}(z)$. Then:

$\sum_{k \geq 0} Y(l-k) X(k)=\sum_{k \geq 0, m>0} Y(l-k) a(m-k) a(-m)-\sum_{k \geq 0, m \geq 0} Y(l-k) a(m) a(-m-k)$.

$\sum_{k \geq 0, m>0} Y(l-k) a(m-k) a(-m) \xi$ is a sum of $2^{(n-1)}$ of expressions of the form

$$
\sum_{k \geq 0, m>0, m_{1}, \ldots, m_{2 n-2}} a\left(m_{1}\right) \ldots a\left(m_{2 n-2}\right) a(m-k) a(-m) \xi
$$

where $0 \leq m_{i} \leq e^{\prime}(1+|\mu-k|+|l-k|)$ by induction hypothesis, and $e^{\prime} \geq 1$ depends only on $Y(z)$. Note that the above expression is nonzero only if $0<m \leq \mu, 0 \leq$ $k \leq \mu$. It follows that the expression is the sum of

$$
\sum_{m_{1}, \ldots, m_{2 n}} a\left(m_{1}\right) \ldots a\left(m_{2 n}\right) \xi
$$

with $0 \leq\left|m_{i}\right| \leq e(1+|l|+\mu), i=1, \ldots, 2 n$, where $e=4 e^{\prime}$. The same conclusion holds for $\sum_{k \geq 0, m>0, m_{1}, \ldots, m_{2 n-2}} a\left(m_{1}\right) \ldots a\left(m_{2 n-2}\right) a(m) a(-m-k) \xi$.

Suppose $V(z)=X^{+}(z) Y(z)$. Then

$\sum_{k<0} X(k) Y(l-k)=\sum_{k<0, m>0} a(m-k) a(-m) Y(l-k)-\sum_{k<0, m \geq 0} a(m) a(-m-k) Y(l-k)$.

$\sum_{k<0, m>0} a(m-k) a(-m) Y(l-k) \xi$ is a sum of $2^{(n-1)}$ of expressions of the form

$$
\sum_{k<0, m>0, m_{1}, \ldots, m_{2 n-2}} a(m-k) a(-m) a\left(m_{1}\right) \ldots a\left(m_{2 n-2}\right) \xi
$$

where $0 \leq m_{i} \leq e^{\prime \prime}(1+|\mu|+|l-k|)$ by induction hypothesis, and $e^{\prime \prime} \geq 1$ depends only on $Y(z)$. Note that the above expression is nonzero only if $0<m \leq \mu-l+k, l-\mu<$ $k<0$. It follows that the expression is the sum of

$$
\sum_{m_{1}, \ldots, m_{2 n}} a\left(m_{1}\right) \ldots a\left(m_{2 n}\right) \xi
$$


with $0 \leq\left|m_{i}\right| \leq \tilde{e}(1+|l|+\mu), i=1, \ldots, 2 n$, where $\tilde{e}=4 e^{\prime \prime}$. The same conclusion holds for $\sum_{k<0, m \geq 0, m_{1}, \ldots, m_{2 n-2}} a(m) a(-m-k) a\left(m_{1}\right) \ldots a\left(m_{2 n-2}\right) \xi$. By induction (1) is proved. Since the norm of $a(m)$ is 1 , it follows from (1) that:

$$
\|V(l) \xi\|_{s} \leq c(1+|\mu-l|)^{s}(1+\mu+|l|)^{a}\|\xi\|,
$$

where $c, a$ are independent of $\xi$ and $l$. Note $(1+|\mu-l|)^{s}(1+\mu+|l|)^{a} \leq(1+$ $\mu)^{s+a}(1+|l|)^{s+a}$ when $s \geq 0$. When $s<0$, the function

$$
\left(\frac{1+x}{1+|x-l|}\right)^{-s}
$$

has maximum $(1+l)^{-s}$ when $x \geq 0, l \geq 0$, and 1 when $x \geq 0, l<0$. It follows that if $s<0$,

$$
(1+|\mu-l|)^{s}(1+\mu+|l|)^{a} \leq(1+\mu)^{s+a}(1+|l|)^{-s+a} .
$$

So we have:

$$
\|V(l) \xi\|_{s} \leq c(1+|l|)^{|s|+a}|| \xi \|_{s+a} .
$$

Now let us consider the case when $G$ is simple and level $k>1$. On $F^{\otimes k}$, $X_{i}^{+} \ldots X_{j}^{+} X_{i^{\prime}}^{-} \ldots X_{j^{\prime}}^{-}$is a summation of $k^{n}$ of expressions of the form: $A:=Y_{1} \otimes$ $Y_{2} \ldots \otimes Y_{l}$, and each $Y_{i}$ is of the form $X_{p}^{+} \ldots X_{q}^{+} X_{p^{\prime}}^{-} \ldots X_{q^{\prime}}^{-}$. Let $\xi_{1} \otimes \ldots \otimes \xi_{k}$ be a vector in $F^{\otimes k}$ with $D \xi_{i}=\mu_{i} \xi_{i}, i=1, \ldots, k$. Then:

$$
\begin{aligned}
& \left\|A(l) \xi_{1} \otimes \ldots \otimes \xi_{k}\right\|_{s}=\left\|\sum_{m_{1}, \ldots, m_{k}, \sum_{i} m_{i}=l} Y_{1}\left(m_{1}\right) \xi_{1} \otimes \ldots \otimes Y_{k}\left(m_{k}\right) \xi_{k}\right\|_{s} \\
& \leq\left(1+\left|\mu_{1}+\ldots+\mu_{k}-l\right|\right)^{s} \sum_{m_{1}, \ldots, m_{k}, \sum_{i} m_{i}=l}\left\|Y_{1}\left(m_{1}\right) \xi_{1} \otimes \ldots \otimes Y_{k}\left(m_{k}\right) \xi_{k}\right\| \\
& =\left(1+\left|\mu_{1}+\ldots+\mu_{k}-l\right|\right)^{s} \sum_{m_{1}, \ldots, m_{k}, \sum_{i} m_{i}=l, m_{i} \leq \mu_{i}}\left\|Y_{1}\left(m_{1}\right) \xi_{1} \otimes \ldots \otimes Y_{k}\left(m_{k}\right) \xi_{k}\right\| \\
& \leq\left(1+\mu_{1}+\ldots+\mu_{k}\right)^{s}(1+|l|)^{|s|} \times c^{\prime}\left(1+\left|m_{1}\right|\right)^{b^{\prime}} \ldots\left(1+\left|m_{k}\right|\right)^{b^{\prime}}\left\|\xi_{1}\right\|_{a^{\prime}} \ldots\left\|\xi_{k}\right\|_{a^{\prime}} \\
& \sum_{m_{1}, \ldots, m_{k}, \sum_{i} m_{i}=l, m_{i} \leq \mu_{i}} \\
& \leq c^{\prime}(1+|l|)^{|s|+b^{\prime} k+k}|| \xi_{1} \otimes \ldots \otimes \xi_{k} \mid \|_{s+b^{\prime} k+k+a^{\prime} k}
\end{aligned}
$$

where in the second $\leq$ we use the result for $k=1$, and in the last $\leq$ we used $l-\sum_{j \neq i} \mu_{j} \leq m_{i} \leq \mu_{i}, i=1, \ldots, k$. The constants $a^{\prime}, b^{\prime}, c^{\prime}$ above are independent of $l, \xi_{1}, \ldots, \xi_{k}$.

Assume $G=G_{1} \times \ldots \times G_{m}$ where $G_{i}, i=1,2, \ldots, m$ are simple factors of $G$. Let $F_{i}$ be the level 1 vacuum fermionic representation of $L G_{i}, i=1, \ldots, m$. On $F_{1}^{k_{1}} \otimes \ldots \otimes F_{m}^{k_{m}}, X_{i}^{+} \ldots X_{j}^{+} X_{i^{\prime}}^{-} \ldots X_{j^{\prime}}^{-}$is still a finite sum of expressions of the form: $A:=Y_{1} \otimes Y_{2} \ldots \otimes Y_{l}$, and each $Y_{i}$ is of the form $X_{p}^{+} \ldots X_{q}^{+} X_{p^{\prime}}^{-} \ldots X_{q^{\prime}}^{-}$. Similar argument 
as in the previous paragraph shows that the lemma is true for such expressions. Since level $\left(k_{1}, \ldots, k_{m}\right)$ vacuum representation of $L\left(G_{1} \times G_{2} \times \ldots \times G_{m}\right)$ appears as a direct summand of $F_{1}^{k_{1}} \otimes \ldots \otimes F_{m}^{k_{m}}$, we have proved the lemma.

By (1) of lemma 1 the domain of $\pi^{0}(V(f))$ can be extended to $H_{\infty}^{0}$, and $\pi^{0}(V(f))$ can be defined for smooth $f$. When no confusion arises, we will denote $\pi^{0}(V(f))$ simply by $V(f)$.

Recall that an operator (not necessarily bounded) $a$ is called affiliated with a von Neumann algebra $M$ if $U^{*} a U=a$ for any unitary $U \in M^{\prime}$ (cf. P. 16 of [Dix]). Our goal is to show that $V(f)$ is affiliated with certain von Neumann algebra (cf. Prop. 2.3 below).

Lemma 2. Assume $f$ is a smooth function. Then:

(1) Denote by $V(f)^{*}$ the adjoint of $V(f)$, then $H_{\infty}^{0}$ is in the domain of $V(f)^{*}$.

(2) The operator $V(f)$ is closable and (we will denote the closure of $V(f)$ by the same symbol) the space of finite energy vectors $H_{0}^{0}$ is a core for $V(f)$;

(3) Suppose $M$ is a von Neumann algebra on $H^{0}$ with a generating self adjoint subset $S \subset M$,i.e., $S^{*}=S$ and the $C^{*}$ algebra generated by $S$ is dense in $M$ in strong topology. If

$$
V(f) s x=s V(f) x, \forall s \in S, x \in H_{0}^{0}
$$

then $V(f)$ is affiliated with $M^{\prime}, j=1,2$.

Proof. Ad (1): It is enough to show that for any $y \in H_{\infty}^{0}$, there exists $w \in H^{0}$ such that

$$
\langle V(f) x, y\rangle=\langle x, w\rangle, \forall x \in H_{\infty}^{0}
$$

Note that if $x \in H_{\infty}^{0}$, then there exists a sequence of finite energy vectors $x_{n}$ such that

$$
\lim _{n \rightarrow \infty}\left\|x_{n}-x\right\|_{a}=0
$$

where $a>0$ is as in lemma 1. By lemma 1

$$
\lim _{n \rightarrow \infty}\left\|V(f) x_{n}-V(f) x\right\|_{a}=0 .
$$

So if

$$
\left\langle V(f) x_{n}, y\right\rangle=\left\langle x_{n}, w\right\rangle, \forall x_{n}
$$

then

$$
\langle V(f) x, y\rangle=\langle x, w\rangle, \forall x \in H_{\infty}^{0}
$$

So we just have to prove the above equation for $x \in H_{0}^{0}$.

First we consider the case that $f$ is a finite energy function. Since $y \in H_{\infty}^{0}$, we can choose a sequence $y_{m}$ such that

$$
\lim _{m \rightarrow \infty}\left\|y_{m}-y\right\|_{a}=0
$$


where $a>0$ is as in lemma 1. By definition we have

$$
\left\langle V(f) x, y_{m}\right\rangle=\left\langle x, V(f)^{F A} y_{m}\right\rangle,
$$

and by (2) of lemma 1

$$
\left\|V(f)^{F A}\left(y_{m}-y_{m^{\prime}}\right)\right\| \leq c\|f\|_{a}\left\|y_{m}-y_{m^{\prime}}\right\|_{a}
$$

So $\left\{V(f)^{F A} y_{m}\right\}_{m \geq 0}$ is a Cauchy sequence with a limit defined to be $V(f)^{F A} y$. We can choose $w=V(f)^{F A} y$. Also note that

$$
\left\|V(f)^{F A} y\right\| \leq c\|f\|_{a}\|y\|_{a} .
$$

Now let $f$ be a smooth function, and choose a sequence $f_{n}$ of finite energy functions such that

$$
\lim _{n \rightarrow \infty}\left\|f_{n}-f\right\|_{a}=0
$$

where constant $a$ is as in lemma 1 . By the proof in the finite energy function case we have

$$
\left\langle V\left(f_{n}\right) x, y\right\rangle=\left\langle x, V\left(f_{n}\right)^{F A} y\right\rangle .
$$

By lemma 1 and the note above

$$
\left\|V\left(f_{n}-f\right) x\right\| \leq c\left\|f_{n}-f\right\|_{a}\|x\|_{a},\left\|V\left(f_{n}-f_{n^{\prime}}\right)^{F A} y\right\| \leq c\left\|f_{n}-f_{n^{\prime}}\right\|\left\|_{a}\right\| y \|_{a} .
$$

It follows that the sequnce $V\left(f_{n}\right)^{F A} y, \forall n>0$ is a Cauchy sequence with a limit denoted by $w$ and

$$
\langle V(f) x, y\rangle=\langle x, w\rangle, \forall x \in H_{0}^{0} .
$$

Ad (2): By (1) $V(f)$ is closable. Let $x \in H_{\infty}^{0}$. Then one can find $x_{n} \in H^{0}$ such that $\left\|x_{n}-x\right\|_{a} \rightarrow 0$ with $a>0$ as in lemma 1 , and by lemma $1 V(f) x_{n} \rightarrow V(f) x$. This shows $H_{0}^{0}$ is a core for $V(f)$.

Ad (3): Suppose $y_{n} \rightarrow y \in M$ in the strong topology and

$$
V(f) y_{n} x=y_{n} V(f) x, \forall s \in S,
$$

and for all $x \in$ the domain of $V(f)$, it follows immediately that $y x$ is in the domain of $V(f)$ and

$$
V(f) y x=y V(f) x, \forall s \in S,
$$

and for all $x \in$ the domain of $V(f)$.

Since

$$
V(f) s x=s V(f) x, \forall s \in S, x \in H_{0}^{0},
$$

and $H_{0}^{0}$ is a core for $V_{j}(f)$, it follows that

$$
V(f) s x=s V(f) x, \forall s \in S,
$$

and for all $x \in$ the domain of $V(f)$. So

$$
V(f) s_{1} s_{2} \ldots s_{n} x=s_{1} s_{2} \ldots s_{n} V(f) x, \forall s_{i} \in S, i=1, \ldots, n
$$

and for all $x \in$ the domain of $V(f)$ and finite $n$. (3) now follows from the definition.

Suppose $p^{*}=-p$ is a smooth test function. Assume that $X \in g$. It follows from $\S 3$ of [GW] (also cf. P. 489 of [W2]) that $X(p)$ is essentially skew-self adjoint with core $H_{0}^{0}$, and $X(p)$ maps $H_{\infty}^{0}$ to $H_{\infty}^{0}$. 
Lemma 3. Let $X \in g$. Then:

(1)

$$
[X(p), V(\psi, f)] x=\sum_{0 \leq j \leq n} V\left(X(j) \psi, \frac{1}{j !} \frac{d^{j} p}{d z^{j}} f(z)\right) x
$$

for any smooth functions $p, f$ and $x \in H_{\infty}^{0}$, where $n$ is the energy of $\psi$;

(2) If $\exp (t X(p)) H_{0}^{0} \subset H_{\infty}^{0},-1 \leq t \leq 1$, and $\operatorname{Sup}\left\{\|\exp (t X(p)) x\|_{s},-1 \leq t \leq\right.$ $1\}<\infty$ for any $x \in H_{0}^{0}, s>0$, then

$\langle[\exp (X(p)), V(\psi, f)] x, y\rangle=\int_{0}^{1}\langle\exp (t X(p))[X(p), V(\psi, f)] \exp ((1-t) X(p)) x, y\rangle d t$

for any smooth functions $p=-p^{*}, f$ and $x \in H_{0}^{0}, y \in H_{0}^{0}$.

Proof. Note for $x, y \in H_{0}^{0},<X(k) V(\psi, z) x-V(\psi, z) X(k) x, y>$ is a polynomial in $z, z^{-1}$.

We have (cf. for an example P. 327 of $[\mathrm{KT}]$ ): for $z \neq 0$,

$$
\begin{aligned}
<X(k) V(\psi, z) x-V & (\psi, z) X(k) x, y>=\frac{1}{2 \pi i} \int_{C_{z}} d w w^{k}<X(w) V(\psi, z) x, y> \\
& =\frac{1}{2 \pi i} \int_{C_{z}} d w w^{k} \sum_{m}(w-z)^{-m-1}<V(X(m) \psi, z) x, y> \\
& =\frac{1}{2 \pi i} \int_{C_{z}} d w w^{k} \sum_{m \leq n}(w-z)^{-m-1}<V(X(m) \psi, z) x, y> \\
& =\sum_{0 \leq j \leq n} \frac{1}{j !} \frac{d^{j} z^{k}}{d z^{j}}<V(X(j) \psi, z) x, y>
\end{aligned}
$$

where $C_{z}$ is the boundary of a disk centered at $z$ with radius $\frac{1}{2}|z|$, and in the last equation we used the fact that

$$
\frac{1}{2 \pi i} \int_{C_{z}} d w w^{k}(w-z)^{-j-1}=\frac{1}{j !} \frac{d^{j} z^{k}}{d z^{j}}
$$

for $0 \leq j \leq n$. Since $H_{0}^{0}$ is dense, we have:

$$
\begin{aligned}
{[X(k), V(\psi, f)] x } & =\sum_{0 \leq j \leq n} \frac{1}{2 \pi i} \int_{S^{1}} \frac{1}{j !} \frac{d^{j} z^{k}}{d z^{j}} f(z) V(X(j) \psi, z) \\
& =\sum_{0 \leq j \leq n} V\left(X(j) \psi, \frac{1}{j !} \frac{d^{j} z^{k}}{d z^{j}} f(z)\right) x
\end{aligned}
$$

which is true for any finite energy function $f$ and $x \in H_{0}^{0}$, and so it is true for any smooth function $f$ and $x \in H_{\infty}^{0}$ by using approximation and lemma 1 . Let $p=\sum_{k} p(k) z^{k}$ be a finite energy function and $x \in H_{\infty}^{0}$. By definition

$$
X(p)=\sum_{k} p(k) X(k),
$$


and so (remember $\left.p=\sum_{k} p(k) z^{k}\right)$

$$
[X(p), V(\psi, f)] x=\sum_{0 \leq j \leq n} V\left(X(j) \psi, \frac{1}{j !} \frac{d^{j} p}{d z^{j}} f(z)\right) x
$$

By lemma 1 the above is also true for any smooth function $p$ since we can always choose a sequence of functions $p_{m}$, each $p_{m}$ is a finite energy function, and $\| p_{m}-$ $p \|_{s} \rightarrow 0$ as $m \rightarrow \infty$ for $s$ greater than a given number which may depend on $X, \psi, X(j) \psi, j=0, \ldots, n$.

Ad (2): Note $H_{\infty}^{0}$ is a subset of $C^{\infty}$ vectors of $X(p)$ and $V(f):=V(\psi, f)$. Let us check that the map

$$
s \in[0,1] \rightarrow A(s):=\langle\exp (s X(p)) V(f) \exp ((1-s) X(p)) x, y\rangle
$$

is a differentiable function with continuous derivative

$$
B(s):=\langle\exp (s X(p))[X(p), V(f)] \exp ((1-s) X(p)) x, y\rangle .
$$

Define

$$
C(s, t):=\langle\exp (s X(p)) V(f) \exp ((1-t) X(p)) x, y\rangle,(s, t) \in[0,1] \times[0,1]
$$

We shall repeatedly use the following elementary fact about $C^{\infty}$ vectors (cf. P. 488 of [W2]): if $\xi$ is a $C^{\infty}$ vector of $X(p)$, i.e., $\xi$ is in the domain of $X(p)^{n}, \forall n \geq 1$, then the function

$$
u \in \mathbb{R} \rightarrow\langle\exp (u X(p)) \xi, \eta\rangle
$$

is a smooth function of $u$ for any $\eta \in H^{0}$.

Since $V(f) \exp ((1-t) X(p)) x \in H_{\infty}^{0}$ is a subset of $C^{\infty}$ vectors of $X(p)$, it follows that $C(s, t)$ is a smooth function of $s$ for fixed $t$. Also note

$$
\begin{aligned}
C(s, t) & =\langle\exp (s X(p)) V(f) \exp ((1-t) X(p)) x, y\rangle \\
& =\left\langle\exp ((1-t) X(p)) x, V(f)^{*} \exp (-s X(p)) y\right\rangle
\end{aligned}
$$

where we have used (1) of lemma 2 and our assumption that $\exp (-s X(p)) y \in$ $H_{\infty}^{0}, 0 \leq s \leq 1$. So $C(s, t)$ is a smooth function of $t$ for fixed $s$. We have ( use (1) of lemma 2 when computing derivatives with respect to $t$ ) the following partial derivatives:

$$
\begin{aligned}
C_{s}(s, t) & =\langle\exp (s X(p)) X(p) V(f) \exp ((1-t) X(p)) x, y\rangle \\
C_{s s}(s, t) & =\left\langle\exp (s X(p)) X(p)^{2} V(f) \exp ((1-t) X(p)) x, y\right\rangle \\
C_{t}(s, t) & =\langle\exp (s X(p)) V(f)(-X(p)) \exp ((1-t) X(p)) x, y\rangle \\
C_{t t}(s, t) & =\left\langle\exp (s X(p)) V(f) X(p)^{2} \exp ((1-t) X(p)) x, y\right\rangle \\
C_{s t}(s, t)=C_{t s}(s, t) & =\langle\exp (s X(p)) X(p) V(f)(-X(p)) \exp ((1-t) X(p)) x, y\rangle
\end{aligned}
$$


Note that all the derivatives above are smooth functions of one variable when the other variable is fixed. We have

$$
\begin{aligned}
\left|C_{s s}(s, t)\right| & \leq\left\|\exp (s X(p)) X(p)^{2} V(f) \exp ((1-t) X(p)) x\right\|\|y\| \\
& \leq\left\|X(p)^{2} V(f) \exp ((1-t) X(p)) x\right\|\|y\| \\
& \leq C_{1}\|p\|_{a_{1}}^{2}\|f\|_{a_{2}}\|\exp ((1-t) X(p)) x\|_{a_{3}}\|y\| \\
& \leq C_{2}
\end{aligned}
$$

where we used lemma 1 in the third $\leq$, the assumption in the last $\leq$, and

$$
C_{1}, C_{2}, a_{1}, a_{2}, a_{3}
$$

are independent of $s, t$ by lemma 1 and the assumption. We can obtain similar estimates for other second partial derivatives, and so there exists a constant $C^{\prime}>0$ such that

$$
\operatorname{Sup}\left\{\left|C_{s s}(s, t)\right|,\left|C_{s t}(s, t)=C_{s t}(t, s)\right|,\left|C_{t t}(s, t)\right|, \forall(s, t) \in[0,1] \times[0,1]\right\} \leq C^{\prime} .
$$

By using the uniform bound for second partial derivatives and Taylor's theorem in calculus, we have

$$
\begin{aligned}
& A(s+\Delta s)-A(s)=C(s+\Delta s, s+\Delta s)-C(s, s) \\
& =C(s+\Delta s, s+\Delta s)-C(s+\Delta s, s)+C(s+\Delta s, s)-C(s, s) \\
& =C_{t}(s+\Delta s, s) \Delta s+\frac{1}{2} C_{t t}\left(s+\Delta s, \theta_{1}\right)(\Delta s)^{2}+C_{s}(s, s) \Delta s+\frac{1}{2} C_{s s}\left(\theta_{2}, s\right)(\Delta s)^{2} \\
& =\left(C_{t}(s, s)+\frac{1}{2} C_{t s}\left(\theta_{3}, s\right) \Delta s\right) \Delta s+\frac{1}{2} C_{t t}\left(s+\Delta s, \theta_{1}\right)(\Delta s)^{2}+ \\
& C_{s}(s, s) \Delta s+\frac{1}{2} C_{s s}\left(\theta_{2}, s\right)(\Delta s)^{2} \\
& =B(s) \Delta s+O\left((\Delta s)^{2}\right)
\end{aligned}
$$

where $\theta_{i}, i=1,2,3$ are between $s$ and $s+\Delta s$,

$$
\left|O\left((\Delta s)^{2}\right)\right| \leq \frac{3 C^{\prime}}{2}\left|(\Delta s)^{2}\right|
$$

and we have used

$$
B(s)=C_{t}(s, s)+C_{s}(s, s)
$$

which follows from definitions. It follows immediately that the derivative of $A(s)$ is $B(s)$ on $[0,1]$. A similar elementary exercise in calculus as above shows that $B(s)$ is continuous on $[0,1]$. (2) now follows by the Fundamental Theorem of Calculus.

Let $T$ be the maximal torus of $G$ and $T=\operatorname{Lie}(T)$. By $\S 13.3$ of $[\mathrm{PS}]$, the level 1 vacuum representation of $L T$ on Hilbert space $F$, is also an irreducible representation of $L G$. Denote by $\pi$ the representation of $L G$ on $F$. 
Lemma 4. (1) Let $k \in \mathbb{N}$ and $x \in F_{0}$, where $F_{0}$ denotes the set of finite energy vectors and $v=\exp (w(p))$ with $w \in \top, p=-p^{*},\|p\|_{k+1}<M$. Then there exists a constant $C$ which only depends on $w, M, k$ and $x \in F_{0}$ such that

$$
\|\pi(v) x\|_{k} \leq C
$$

(2) Let $u=\exp (X(p))$ where $X=X_{\alpha}$ or $X=Y_{\alpha}$ and $p=-p^{*},\|p\|_{k+1}<M$. Then then there exists a constant $C^{\prime}$ which only depends on $X, M, k$ and $x \in F_{0}$ such that

$$
\|\pi(u) x\|_{k} \leq C^{\prime}
$$

(3) Let $u=\exp (X(p))$ where $X=X_{\alpha}$ or $X=Y_{\alpha}$ and $p=-p^{*},\|p\|_{k+1}<M$. Denote by $\pi^{0}$ the vacuum representation of $L G$ on $H^{0}$. Then then there exists a constant $C^{\prime \prime}$ which only depends on $X, M, k$ and $x \in H_{0}^{0}$ such that

$$
\left\|\pi^{0}(u) x\right\|_{k} \leq C^{\prime \prime}
$$

Proof. Ad (1): The basic idea is contained in Prop. 9.5.15 of [PS] and we will recall the notations and facts in $9.5[\mathrm{PS}]$. We can write $L T \simeq \operatorname{Hom}\left(S^{1}, T\right) \times T \times V$, where $T$ is the subgroup of constant loops, and $V$ is the vector space of maps $a: S^{1} \rightarrow \top$ with integral 0 , which is regarded as a subgroup of $L T$ by the exponential map $a \rightarrow \exp (i a)$. The identity component of the central extension of $L T$ in our case is canonically a product $T \times \tilde{V}$, where $\tilde{V}$ is the Heisenberg group associated to a skew form $S$ defined on P. 63 of [PS]. Write $V \otimes \mathbb{C}=A \oplus \bar{A}$, where $A$ is spanned by $z^{k} \top \otimes \mathbb{C}$ for $k>0$. For $a \in V \otimes \mathbb{C}$, let $a=\sum_{n} a_{n} z^{n}, a_{n} \in \top \otimes \mathbb{C}$ be its Fourier series. We define

$$
\|a\|_{s}:=\sum_{n}(1+|n|)^{s}\left|a_{n}\right|, s \in \mathbb{R}
$$

and $|$.$| is the norm on T \otimes \mathbb{C}$ induced from the restriction of Killing form on $T$. The Hermitian form $\langle$,$\rangle on A$ defined by

$$
\left\langle a, a^{\prime}\right\rangle=-2 i S(\bar{\xi}, \eta)
$$

is positive definite, where the skew form $S$ is defined on P. 63 of [PS]. The only property we need about $S$ is

$$
\left|S\left(a, a^{\prime}\right)\right| \leq\|a\|_{0}\left\|a^{\prime}\right\|_{1}, \forall a, a^{\prime} \in V
$$

which follows from its definition.

The Hilbert space $F$ is the completion of the symmetric algebra $S(A)$ with respect to Hermitian form above, which is extended from $A$ to $S(A)$ by the formula

$$
\left\langle a_{1} a_{2} \ldots a_{n}, a_{1}^{\prime} a_{2}^{\prime} \ldots a_{n}^{\prime}\right\rangle=\sum\left\langle a_{1}, a_{i_{1}}^{\prime}\right\rangle \ldots\left\langle a_{n}, a_{i_{n}}^{\prime}\right\rangle
$$


where the sum is over all permutations $\left\{i_{1}, \ldots, i_{n}\right\}$ of $\{1, \ldots, n\}$. Note that for any $a \in A$,

$$
e^{a}:=\sum_{n \geq 0} \frac{a^{n}}{n !}
$$

belongs to $F$. The vacuum vector in $F$ is denoted by 1 .

As in the proof of Prop. 9.5.15 of [PS], it is sufficient to prove (1) for the case when $v \in \tilde{V}$ and $x$ is the vacuum vector. Note that $v=\exp (w(p))$ is identified with $\tilde{w}(p)=i p w \in \tilde{V}$ under the isomorphism $L T \simeq \operatorname{Hom}\left(S^{1}, T\right) \times T \times V$ above.

The action of $v$ on vacuum vector 1 is given by (cf. P. 194 of [PS]):

$$
v .1=e^{-\frac{1}{2}\langle a, a\rangle} e^{a},
$$

where $\tilde{w}(p)=a+\bar{a}$, and $a(z)=\sum_{i>0} a_{i} z^{i}$. Let

$$
a^{(s)}(z):=\sum_{i>0} i^{s} a_{i} z^{i}, s \in \mathbb{N} .
$$

Note that $\left\|a^{(s)}\right\|_{0} \leq\|a\|_{s} \leq\|p\| \|_{s}|w|$. We have:

$$
D^{k} a^{n}=\sum_{s_{1} \geq 0, \ldots s_{n} \geq 0, s_{1}+\ldots+s_{n}=k} \frac{k !}{s_{1} ! \ldots s_{n} !} a^{\left(s_{1}\right)} \ldots a^{\left(s_{n}\right)},
$$

and so

$$
\left\|D^{k} a^{n}\right\| \leq \sum_{s_{1} \geq 0, \ldots s_{n} \geq 0, s_{1}+\ldots+s_{n}=k} \frac{k !}{s_{1} ! \ldots s_{n} !}\left\|a^{\left(s_{1}\right)} \ldots a^{\left(s_{n}\right)}\right\| .
$$

Note that for $0 \leq s_{1}, t_{1} \leq k$,

$$
\begin{aligned}
\left|\left\langle a^{\left(s_{1}\right)}, a^{\left(t_{1}\right)}\right\rangle\right| & =\left|2 S\left(a^{\left(s_{1}\right)}, \overline{a^{\left(t_{1}\right)}}\right)\right| \leq 2\left\|a^{\left(s_{1}\right)}\right\|\left\|_{0}\right\| a^{\left(t_{1}\right)} \|_{1} \\
& \leq 2\|a\|_{s_{1}}\|a\|_{t_{1}+1} \leq 2\|p\|_{k+1}^{2}|w|^{2}
\end{aligned}
$$

hence

$$
\begin{aligned}
\left\|a^{\left(s_{1}\right)} \ldots a^{\left(s_{n}\right)}\right\|^{2} & =\left\langle a^{\left(s_{1}\right)} \ldots a^{\left(s_{n}\right)}, a^{\left(s_{1}\right)} \ldots a^{\left(s_{n}\right)}\right\rangle \\
& \leq n ! 2^{n}|w|^{2 n}\|p\|_{k+1}^{2 n}
\end{aligned}
$$

where we used the definition of $\langle$,$\rangle on F$ as completion of $S(A)$. So

$$
\left\|D^{k} a^{n}\right\| \leq(n !)^{\frac{1}{2}}\|p\|_{k+1}^{n} n^{k}(\sqrt{2}|w|)^{n},
$$

and

$$
\begin{aligned}
\left\|D^{k} v .1\right\| & \leq e^{-\frac{1}{2}\langle a, a\rangle} \sum_{n=1}^{\infty} \frac{1}{(n !)^{\frac{1}{2}}}\|p\|_{k+1}^{n} n^{k}(\sqrt{2}|w|)^{n} \\
& \leq \sum_{n=1}^{\infty} \frac{1}{(n !)^{\frac{1}{2}}}\|p\|_{k+1}^{n} n^{k}(\sqrt{2}|w|)^{n} .
\end{aligned}
$$


This implies (1) by the definition of $\|.\|_{k}$.

Ad (2): By the observation on Page 267 of $[\mathrm{PS}]$ there exists an element $q \in G$ such that

$$
q \exp (v(p)) q^{-1}=\exp (X(p))
$$

up to a scalar as operators on $F$. Since the action of $q$ commutes with the action of rotation, (2) follows from (1).

Ad (3): It is enough to consider the case when $G$ is simple. Since any level $L$ vacuum representation appears as a direct summand of $F^{\otimes L}$, we just have to prove (3) for the representation $\pi^{\otimes L}$, but this follows immediately from (2).

Lemma 5. (1)

If $f, p=-p^{*}$ are smooth functions, then

$$
\pi^{0}(\exp (X(p))) \pi^{0}(V(\psi, f)) x=\pi^{0}(V(\psi, f)) \pi^{0}(\exp (X(p))) x
$$

for any $x \in H_{0}^{0}, X \in \operatorname{Lie}(H)$;

(2) Let $f, p=-p^{*}$ be smooth functions on $S^{1}$ with support $f \subset I$ and support $p \subset I^{\prime}$. If $X=X_{\alpha}$ or $X=Y_{\alpha}$, then:

$$
\pi^{0}(V(\psi, f)) \pi^{0}(\exp (X(p))) x=\pi^{0}(\exp (X(p))) \pi^{0}(V(\psi, f)) x
$$

for any $x \in H_{0}^{0}$.

Proof. Ad (1): If $X \in \operatorname{Lie}(H), X(i) \psi=0$ for any $i \geq 0$ by the definition of $\psi$, it follows from (1) of lemma 3 that

$$
[X(p), V(\psi, f)] x=0
$$

for any $x \in H_{\infty}^{0}$. By (3) of lemma 4, the condition of (2) of lemma 3 (note in (2) of lemma $3 t X(p)=X(t p)$ by definition) is satisfied, and the identity follows by using (2) of lemma 3 and the fact that $H_{0}^{0}$ is norm dense in $H^{0}$.

Ad (2): Since the support of $f$ and the support of $p$ are disjoint, by (1) of lemma 3

$$
[X(p), V(\psi, f)] x=0
$$

for any $x \in H_{\infty}^{0}$. By (3) of lemma 4 , the condition of (2) of lemma 3 is satisfied, and the identity follows by using (2) of lemma 3 and the fact that $H_{0}^{0}$ is norm dense in $H^{0}$. 
Lemma 6. Let $S$ be the set which consists of elements $\pi^{0}(\exp (X(p)))$ with $p=-p^{*}$ smooth if $X \in \operatorname{Lie}(H)$, and $p=-p^{*}$ smooth, support $p \subset I^{\prime}$ if $X=X_{\alpha}$ or $X=Y_{\alpha}$. Then the $C^{*}$ algebra generated by $S$ is strongly dense in $\pi^{0}(L H)^{\prime \prime} \vee \pi^{0}\left(L_{I^{\prime}} G\right)^{\prime \prime}$ if $H$ is simply connected.

Proof. Note $S=S^{*}$. Since every element of $L_{I^{\prime}} G$ (resp. $L H$ ) is a product of exponentials in $L_{I^{\prime}} g$ (resp. $L h$ ), cf. P. 487 of [W2] (we use the fact that $G, H$ are simply connected here), we just have to show every element of the form $\pi^{0}(\exp (X(p)))$ with $p=-p^{*}$ smooth, support $p \subset I^{\prime}$, and $X \in g$ is in the von Neumann algebra $M$ generated by $S$. Assume $X=\sum_{i} c_{i} X_{i}$, where $c_{i} \in \mathbb{R}$ and $X_{i}$ is either $X_{\alpha}$ or $Y_{\alpha}$. Note that $\pi^{0}(X(p))$ and $\pi^{0}\left(X_{i}(p)\right)$ are essentially skew self-adjoint operator with a common core $H_{0}^{0}$. By abuse of notations, we will use the same symbol to denote its closure. Let $a \in M^{\prime}$. Then we have:

$$
\pi^{0}\left(X_{i}(p)\right) a x=a \pi^{0}\left(X_{i}(p)\right) x
$$

for any $x \in H_{0}^{0}$, so

$$
\pi^{0}(X(p)) a x=a \pi^{0}(X(p)) x
$$

for any $x \in H_{0}^{0}$, and it follows that the closure of

$$
\pi^{0}(X(p))
$$

is affiliated with $M$, so $\pi^{0}(\exp (X(p)))$ is in $M$.

Proposition 2.3. Suppose $H \subset G, H$ is simply connected, and $\pi^{0}$ is the vacuum representation of $L G$. Let $f$ be a smooth function with support $f \subset I$. Then $\pi^{0}(V(\psi, f))$ is affiliated with von Neumann algebra $\pi^{0}(L H)^{\prime} \cap \pi^{0}\left(L_{I} G\right)^{\prime \prime}$.

Proof. By lemma 5,

$$
\pi^{0}(V(\psi, f)) u x=u \pi^{0}(V(\psi, f)) x
$$

for any $u \in S$ where $S$ is the generating set as in lemma 6 and $x \in H_{0}^{0}$. Note by Haag duality in Prop. 2.1

$$
\pi^{0}\left(L_{I} G\right)^{\prime}=\pi^{0}\left(L_{I^{\prime}} G\right)^{\prime \prime}
$$

The proposition now follows from (3) of lemma 2 and lemma 6.

Now we can finish the proof of Th. 2.3.

Proof of Th. 2.3. Let $\psi \in H_{0,0} \otimes \Omega_{0}$ be an eigenvector of $D$ with eigenvalue $n \in \mathbb{N}$. Note $\psi=V(-n) \Omega=V(\psi, p) \Omega$ with $p=z^{-1}$. Choose two smooth functions $f_{1}$ and $f_{2}$, with support $f_{1} \subset I_{1} \in \mathcal{I}$ and support $f_{2} \subset I_{2} \in \mathcal{I}$, and $f_{1}+f_{2}=1$. Then

$$
\psi=V(\psi, p) \Omega=V\left(\psi, p f_{1}\right) \Omega+V\left(\psi, p f_{2}\right) \Omega .
$$


By Prop. 2.3, the closed operator $V(\psi, f)$ is affiliated with $\pi^{0}(L H)^{\prime} \cap \pi^{0}\left(L_{J} G\right)^{\prime \prime}$ if the support $f \subset J \in \mathcal{I}$. Let $V(\psi, f)=U|V|$ be the polar decomposition. By lemma 4.4.1 of $[\mathrm{Mv}], U$ and $\exp (i t|V|), \forall t \in \mathbb{R}$ are in $\pi^{0}(L H)^{\prime} \cap \pi^{0}\left(L_{J} G\right)^{\prime \prime}$. Since $\Omega$ is in the domain of $|V|$, it follows by Stone's theorem (cf. P. 266 of [RS]) that as $t \rightarrow 0$, $\left(e^{i t|V|} \Omega-\Omega\right) / t \rightarrow|V| \Omega$, and this shows

$$
V(\psi, f) \Omega=U|V| \Omega \in \overline{\pi^{0}(L H)^{\prime} \cap \pi^{0}\left(L_{J} G\right)^{\prime \prime} \Omega} \subset \mathcal{H} .
$$

So

$$
V\left(\psi, p f_{1}\right) \Omega \in \mathcal{H}, V\left(\psi, p f_{2}\right) \Omega \in \mathcal{H}
$$

and it follows that $\psi \in \mathcal{H}$ by the expression given at the beginning of the proof.

2.3 Two Conjectures. We will use the notations in 2.1.

Conjecture 1. The covariant representations $\pi_{i, \alpha}$ can be decomposed into a direct sum of a finite number of irreducible representations and the localized sectors corresponding to these irreducible representations generate a finite dimensional ring over $\mathbb{C}$ under the product of sectors (cf. 4.1).

Conjecture 1 comes from the physicists' argument that the coset $H \subset G$ CFT is a rational CFT: there are only a finite number of primary fields. Here the primary fields correspond to the representations or sectors. In some cases, e.g., $G \subset G \times G$ where the inclusion is diagonal, there are also conjectures on the structure constants of the ring (cf. [FKW] and [BBSS]). More precisely the conjectures in $\S 3$ and 4 of $[\mathrm{FKW}]$ are about certain representations of $\mathrm{W}$-algebras with critical parameters. The W-algebras defined in [FKW] are closed related to the coset $G \subset G_{1} \times G_{m}$ (cf: [Watts]), for an example, the representations of W-algebras in [FKW] have the same characters as those which come from the coset. We shall call the irreducible conformal precosheaves of the cosets $S U(N) \subset S U(N)_{1} \times S U(N)_{m}$ coset $W_{N}$-algebras with critical parameters. Note that coset $W_{2}$ algebras with critical parameters are the irreducible conformal precosheaves corresponding to Virasoro algebras studied in [GKO] and [Luke].

To state conjecture 2, let $L_{0}^{g, h}$ be the generator of rotation group for the coset as in the proof of Prop. 2.2. Then $e^{-\beta L_{0}}, \beta>0$ is a trace-class operator on $H_{i, \alpha}$ by Th. B of $\left[\mathrm{KW}\right.$. Denote by $d_{i, \alpha}$ the statistical dimension (cf. 4.1) of $\pi_{i, \alpha}$. Then we have: (cf. [L5] and (4.27) of [FG])

\section{Conjecture 2 (also known as Kac-Wakimoto formula in [L5]).}

$$
d_{i, \alpha}=\lim _{\beta \rightarrow 0} \frac{\operatorname{Tr}_{H_{i, \alpha}} e^{-\beta L_{0}}}{\operatorname{Tr}_{H_{0,0}} e^{-\beta L_{0}}}
$$

Both of these conjectures are highly nontrivial. The results in [W2] prove these conjectures in the case $G$ is of type $A$ and $H$ is a trivial group. For the case of coset 
$W_{2}$ algebras with critical parameters the above conjectures follow from the results of [Luke]. Note that Conjecture 2 immediately implies Kac-Wakimoto conjecture (cf. Conj. 2.5 in $[\mathrm{KW}]$ ). In fact, Conjecture 2 can also be stated as:

$$
d_{i, \alpha}=\frac{b(i, \alpha)}{b(0,0)}
$$

where $b(i, \alpha)$ is defined as in $\S 2$ of $[\mathrm{KW}]$ with our $(i, \alpha)$ identified with $(\Lambda, \lambda)$ in (2.5.4) of $[\mathrm{KW}$. Conj. 2.5 in $[\mathrm{KW}]$ states that $b(i, \alpha)>0$. Conjecture 2 is stronger than this since $d_{i, \alpha} \geq 1$ and $b(0,0)>0$ by definitions.

Note that the Kac-Wakimoto hypothesis (cf. Page 161 of [KW]) also implies Kac-Wakimoto conjecture, but the first counter-example to Kac-Wakimoto hypothesis has been found in [X2] by considering subfactors associated with conformal inclusions. So far Conjecture 2 and hence the Kac-Wakimoto conjecture have been checked to be true in all known examples.

\section{§3. Commuting Squares}

We will use the notations of 2.1. All the cosets considered in this section are assumed to verify the assumptions of Th. 2.3 unless stated otherwise. For the definitions and properties of statistical dimensions and minimal index, see 4.1.

Definition (cofiniteness). The coset $H \subset G_{L}$ is called cofinite if the inclusion

$$
\left(\pi^{0}\left(L_{I} G\right)^{\prime \prime} \cap \pi^{0}\left(L_{I} H\right)^{\prime}\right) \vee \pi^{0}\left(L_{I} H\right)^{\prime \prime} \subset \pi^{0}\left(L_{I} G\right)^{\prime \prime}
$$

has finite statistical dimension. The statistical dimension of the inclusion is denoted by $d(G / H)$.

Note that $d(G / H)$ does not depend on the choice of $I$ by the covariance property of representations (cf. Prop. 2.1 of $[\mathrm{GL}]$ ), and we can replace $\pi^{0}$ by any level $L$ representation of $L G$ in the above definition due to the local equivalence of these representations (cf. Th. II. B of [W2]).

Let $\pi^{i}$ be an irreducible projective representations of $L G$ with positive energy at level $L$ on Hilbert space $H^{i}$. Recall (cf. 2.1) when restricting to $L H, H^{i}$ decomposes as:

$$
H^{i}=\sum_{\alpha} H_{i, \alpha} \otimes H_{\alpha}
$$

and $\pi_{\alpha}$ are irreducible projective representations of $L H$ on Hilbert space $H_{\alpha}$, and the sum is over $\alpha$ such that $(i, \alpha) \in \exp$. Consider the following inclusions:

$$
\begin{aligned}
& \left(\pi^{i}\left(L_{I} G\right)^{\prime \prime} \cap \pi^{i}\left(L_{I} H\right)^{\prime}\right) \vee \pi^{i}\left(L_{I} H\right)^{\prime \prime} \subset \pi^{i}\left(L_{I} G\right)^{\prime \prime} \subset \pi^{i}\left(L_{I^{\prime}} G\right)^{\prime} \\
& \subset\left(\left(\pi^{i}\left(L_{I^{\prime}} G\right)^{\prime \prime} \cap \pi^{i}\left(L_{I^{\prime}} H\right)^{\prime}\right) \vee \pi^{i}\left(L_{I^{\prime}} H\right)^{\prime \prime}\right)^{\prime}
\end{aligned}
$$

Note that

$$
\pi^{i}\left(L_{I^{\prime}} G\right)^{\prime} \subset\left(\left(\pi^{i}\left(L_{I^{\prime}} G\right)^{\prime \prime} \cap \pi^{i}\left(L_{I^{\prime}} H\right)^{\prime}\right) \vee \pi^{i}\left(L_{I^{\prime}} H\right)^{\prime \prime}\right)^{\prime}
$$


has the same statistical dimension as

$$
\left(\pi^{i}\left(L_{I^{\prime}} G\right)^{\prime \prime} \cap \pi^{i}\left(L_{I^{\prime}} H\right)^{\prime}\right) \vee \pi^{i}\left(L_{I^{\prime}} H\right)^{\prime \prime} \subset \pi^{i}\left(L_{I^{\prime}} G\right)^{\prime \prime}
$$

which is $d(G / H)$. By the multiplicativity of statistical dimensions (cf. 4.1) the statistical dimension of the inclusion

$$
\begin{aligned}
& \left(\pi^{i}\left(L_{I} G\right)^{\prime \prime} \cap \pi^{i}\left(L_{I} H\right)^{\prime}\right) \vee \pi^{i}\left(L_{I} H\right)^{\prime \prime} \subset \\
& \left(\left(\pi^{i}\left(L_{I^{\prime}} G\right)^{\prime \prime} \cap \pi^{i}\left(L_{I^{\prime}} H\right)^{\prime}\right) \vee \pi^{i}\left(L_{I^{\prime}} H\right)^{\prime \prime}\right)^{\prime}
\end{aligned}
$$

is $d_{i} d(G / H)^{2}$, where $d_{i}$ is the statistical dimension of $\pi^{i}\left(L_{I} G\right)^{\prime \prime} \subset \pi^{i}\left(L_{I^{\prime}} G\right)^{\prime}$. On the other hand by the additivity of statistical dimension (cf. 4.1)) the statistical dimension of the above inclusion is $\sum_{\alpha} d_{(i, \alpha)} d_{\alpha}$, where $d_{(i, \alpha)}$ and $d_{\alpha}$ are the statistical dimensions of $\pi_{i, \alpha}$ and $\pi_{\alpha}$ respectively. So we have

$$
d_{i} d(G / H)^{2}=\sum_{\alpha} d_{(i, \alpha)} d_{\alpha}
$$

When any of the statistical dimensions in formula (3.1) are $\infty$, then (3.1) is understood as the statement that both sides of the equation are $\infty$. See the paragraph before Prop. 4.2 for a slightly different derivation of formula (3.1).

When $i=0$ is the vacuum representation, the statistical dimension $d_{0}$ of the inclusion

$$
\pi^{0}\left(L_{I} G\right)^{\prime \prime} \subset \pi^{0}\left(L_{I^{\prime}} G\right)^{\prime}
$$

is 1 by Haag duality in Prop. 2.1. It follows from formula (3.1) that $H \subset G_{L}$ is cofinite if and only if $d_{(0, \alpha)} d_{\alpha}<\infty$ for all $(0, \alpha) \in \exp$. Hence Conjecture 2 implies the cofiniteness for any coset.

For simplicity we will drop the subscript $L$ in the following when no confusion arises. Note that if $H=\{e\}$ is the trivial group, then $H \subset G$ is cofinite. So the statement "if $H_{1} \subset G$ is cofinite and $H_{1} \subset H_{2} \subset G$, then $H_{2} \subset G$ is cofinite" is as difficult to prove as the statement " $H_{2} \subset G$ is cofinite" by simply taking $H_{1}=\{e\}$. But we have:

Proposition 3.1. Suppose $H_{1} \subset H_{2} \subset G$.

(1) If $H_{1} \subset G$ is cofinite, then $H_{1} \subset H_{2}$ is cofinite, and $d\left(G / H_{1}\right) \geq d\left(H_{2} / H_{1}\right)$;

(2) If $H_{1} \subset H_{2}$ and $H_{2} \subset G$ are cofinite, then $H_{1} \subset G$ is cofinite and $d\left(G / H_{1}\right) \leq$ $d\left(G / H_{2}\right) \times d\left(H_{2} / H_{1}\right)$.

This proposition is proved below by using commuting squares. Commuting squares where all the algebras are finite type can be found in reference [We], $\mathrm{Po}]$. But we will consider the case where all the algebras are type $I I I$.

Since the action of the modular group of $\pi^{0}\left(L_{I} G\right)^{\prime \prime}$ with respect to the vacuum vector $\Omega$ is geometric and ergodic (cf. 2.1), it follows from Takesaki's theorem (cf. [MT] or P. 495 of [W2]) that the von Neumann algebras $\pi^{0}\left(L_{I} H_{i}\right)^{\prime \prime} \vee$ 
$\left(\pi^{0}\left(L_{I} H_{i}\right)^{\prime} \cap \pi^{0}\left(L_{I} G\right)^{\prime \prime}\right), \quad i=1,2$ and $\pi^{0}\left(L_{I} H_{1}\right)^{\prime \prime} \vee\left(\pi^{0}\left(L_{I} H_{1}\right)^{\prime} \cap \pi^{0}\left(L_{I} H_{2}\right)^{\prime \prime}\right) \vee$ $\left(\pi^{0}\left(L_{I} H_{2}\right)^{\prime} \cap \pi^{0}\left(L_{I} G\right)^{\prime \prime}\right)$ are factors, and there exist normal faithful conditional expectations $\epsilon_{i}: \pi^{0}\left(L_{I} G\right)^{\prime \prime} \rightarrow \pi^{0}\left(L_{I} H_{i}\right)^{\prime \prime} \vee\left(\pi^{0}\left(L_{I} H_{i}\right)^{\prime} \cap \pi^{0}\left(L_{I} G\right)^{\prime \prime}\right), i=1,2$ and $\epsilon: \pi^{0}\left(L_{I} G\right)^{\prime \prime} \rightarrow \pi^{0}\left(L_{I} H_{1}\right)^{\prime \prime} \vee\left(\pi^{0}\left(L_{I} H_{1}\right)^{\prime} \cap \pi^{0}\left(L_{I} H_{2}\right)^{\prime \prime}\right) \vee\left(\pi^{0}\left(L_{I} H_{2}\right)^{\prime} \cap \pi^{0}\left(L_{I} G\right)^{\prime \prime}\right)$. Moreover, these conditional expectations preserve the state $\omega$ on $\pi^{0}\left(L_{I} G\right)^{\prime \prime}$ defined by $\omega(x)=(x \Omega, \Omega)$, i.e., $\omega(x)=\omega\left(\epsilon^{\prime}(x)\right), \forall x \in \pi^{0}\left(L_{I} G\right)^{\prime \prime}$, when $\epsilon^{\prime}=\epsilon_{1}, \epsilon_{2}, \epsilon$ respectively. Then we have:

Lemma 3.1 (Commuting Square). (1) $\epsilon_{1} \cdot \epsilon_{2}=\epsilon_{1} \cdot \epsilon_{2}=\epsilon$;

(2) $\epsilon_{1}, \epsilon_{2}$ are minimal if $\epsilon$ has finite index.

Proof. Assume the vacuum representation $\pi^{0}$ of $L G$ decomposes with respect to $\mathrm{LH}_{2}$ as:

$$
H^{0}=\oplus_{\alpha} H_{G / H_{2}, 0, \alpha} \otimes H_{H_{2}, \alpha},
$$

then with respect to $L H_{1}$ the decomposition is:

$$
\begin{aligned}
H^{0} & =\oplus_{\alpha} H_{G / H_{2}, 0, \alpha} \otimes H_{H_{2}, \alpha} \\
& =\oplus_{\alpha} H_{G / H_{2}, 0, \alpha} \otimes\left(\oplus_{\beta} H_{H_{2} / H_{1}, \alpha, \beta} \otimes H_{H_{1}, \beta}\right)
\end{aligned}
$$

Let $P_{1}$ be the projection from $H^{0}$ onto

$$
\oplus_{\alpha} H_{G / H_{2}, 0, \alpha} \otimes H_{H_{2} / H_{1}, \alpha, 0} \otimes H_{H_{1}, 0},
$$

$P_{2}$ the projection from $H^{0}$ onto

$$
H_{G / H_{2}, 0,0} \otimes \oplus_{\beta}\left(H_{H_{2} / H_{1}, 0, \beta} \otimes H_{H_{1}, \beta}\right),
$$

and $P$ the projection from $H^{0}$ onto

$$
H_{G / H_{2}, 0,0} \otimes H_{H_{2} / H_{1}, 0,0} \otimes H_{H_{1}, 0} .
$$

It follows from definitions that $P_{1} P_{2}=P_{2} P_{1}=P$. By Th. 2.3 and Reeh-Schlieder Theorem in Prop. 2.1

$$
P_{i} H^{0}=\overline{\pi^{0}\left(L_{I} H_{i}\right)^{\prime \prime} \vee\left(\pi^{0}\left(L_{I} H_{i}\right)^{\prime} \cap \pi^{0}\left(L_{I} G\right)^{\prime \prime}\right) \Omega}, i=1,2,
$$

and

$$
P H^{0}=\overline{\pi^{0}\left(L_{I} H_{1}\right)^{\prime \prime} \vee\left(\pi^{0}\left(L_{I} H_{1}\right)^{\prime} \cap \pi^{0}\left(L_{I} H_{2}\right)^{\prime \prime}\right) \vee\left(\pi^{0}\left(L_{I} H_{2}\right)^{\prime} \cap \pi^{0}\left(L_{I} G\right)^{\prime \prime}\right) \Omega} .
$$

So for any $x \in \pi^{0}\left(L_{I} G\right)^{\prime \prime}$, we have:

$$
\epsilon_{1}\left(\epsilon_{2}(x)\right) \Omega=P_{1} \epsilon_{2}(x) \Omega=P_{1} P_{2} x \Omega=P x \Omega=\epsilon(x) \Omega,
$$

and similarly

$$
\epsilon_{2}\left(\epsilon_{1}(x)\right) \Omega=\epsilon(x) \Omega
$$


Since $\Omega$ is separating for $\pi^{0}\left(L_{I} G\right)^{\prime \prime},(1)$ of lemma is proved.

Note by the remark at the end of 2.2 , the inclusions

$$
\pi^{0}\left(L_{I} H_{i}\right)^{\prime \prime} \vee\left(\pi^{0}\left(L_{I} H_{i}\right)^{\prime} \cap \pi^{0}\left(L_{I} G\right)^{\prime \prime}\right) \subset \pi^{0}\left(L_{I} G\right)^{\prime \prime}
$$

are irreducible, so by Prop. 4.3 of [L4] $\epsilon_{i}$ are unique and must be the minimal conditional expectations, $i=1,2$ if the index of $\epsilon$ is finite.

Proof of Prop. 3.1. (1) As in the proof of lemma 3.1, suppose the vacuum representation $\pi^{0}$ of $L G$ decomposes with respect to $L H_{2}$ as:

$$
H^{0}=\oplus_{\alpha} H_{G / H_{2}, 0, \alpha} \otimes H_{H_{2}, \alpha},
$$

and let $P_{0}$ (resp. $P_{00}$ ) be the projection from $H^{0}$ onto $H_{G / H_{2}, 0,0} \otimes H_{H_{2}, 0}$ (resp. $H_{G / H_{2}, 0,0} \otimes \Omega_{0}$ where $\Omega_{0}$ is the vacuum vector in $\left.H_{H_{2}, 0}\right)$. Then

$$
\begin{aligned}
\pi^{0}\left(L_{I} H_{2}\right)^{\prime \prime} \vee\left(\pi^{0}\left(L_{I} H_{2}\right)^{\prime} \cap \pi^{0}\left(L_{I} G\right)^{\prime \prime}\right) & \simeq \pi^{0}\left(L_{I} H_{2}\right)^{\prime \prime} \vee\left(\pi^{0}\left(L_{I} H_{2}\right)^{\prime} \cap \pi^{0}\left(L_{I} G\right)^{\prime \prime}\right) P_{0} \\
& \simeq \pi_{0}\left(L_{I} H_{2}\right)^{\prime \prime} \otimes\left(\pi^{0}\left(L_{I} H_{2}\right)^{\prime} \cap \pi^{0}\left(L_{I} G\right)^{\prime \prime}\right) P_{00} \\
& \simeq \pi^{0}\left(L_{I} H_{2}\right)^{\prime \prime} \otimes\left(\pi^{0}\left(L_{I} H_{2}\right)^{\prime} \cap \pi^{0}\left(L_{I} G\right)^{\prime \prime}\right)
\end{aligned}
$$

where $\otimes$ is the tensor product of von Neumann algebras and $A \simeq B$ means $A$ and $B$ are ${ }^{*}$-isomorphic, since all the algebras above are factors. Note the $*$-simorphism above from

$$
\pi^{0}\left(L_{I} H_{2}\right)^{\prime \prime} \vee\left(\pi^{0}\left(L_{I} H_{2}\right)^{\prime} \cap \pi^{0}\left(L_{I} G\right)^{\prime \prime}\right)
$$

to $\pi^{0}\left(L_{I} H_{2}\right)^{\prime \prime} \otimes\left(\pi^{0}\left(L_{I} H_{2}\right)^{\prime} \cap \pi^{0}\left(L_{I} G\right)^{\prime \prime}\right)$ maps $\pi^{0}(x)$ to $\pi^{0}(x) \otimes 1, \forall x \in L_{I} H_{2}$, and $y$ to $1 \otimes y, \forall y \in \pi^{0}\left(L_{I} H_{2}\right)^{\prime} \cap \pi^{0}\left(L_{I} G\right)^{\prime \prime}$. So this $*$-simorphism maps

$$
\pi^{0}\left(L_{I} H_{1}\right)^{\prime \prime} \vee\left(\pi^{0}\left(L_{I} H_{1}\right)^{\prime} \cap \pi^{0}\left(L_{I} H_{2}\right)^{\prime \prime}\right) \vee\left(\pi^{0}\left(L_{I} H_{2}\right)^{\prime} \cap \pi^{0}\left(L_{I} G\right)^{\prime \prime}\right)
$$

onto

$$
\left(\pi^{0}\left(L_{I} H_{1}\right)^{\prime \prime} \vee\left(\pi^{0}\left(L_{I} H_{1}\right)^{\prime} \cap \pi^{0}\left(L_{I} H_{2}\right)^{\prime \prime}\right)\right) \otimes\left(\pi^{0}\left(L_{I} H_{2}\right)^{\prime} \cap \pi^{0}\left(L_{I} G\right)^{\prime \prime}\right) .
$$

It follows that the inclusion

$$
\begin{aligned}
& \pi^{0}\left(L_{I} H_{1}\right)^{\prime \prime} \vee\left(\pi^{0}\left(L_{I} H_{1}\right)^{\prime} \cap \pi^{0}\left(L_{I} H_{2}\right)^{\prime \prime}\right) \vee\left(\pi^{0}\left(L_{I} H_{2}\right)^{\prime} \cap \pi^{0}\left(L_{I} G\right)^{\prime \prime}\right) \subset \\
& \pi^{0}\left(L_{I} H_{2}\right)^{\prime \prime} \vee\left(\pi^{0}\left(L_{I} H_{2}\right)^{\prime} \cap \pi^{0}\left(L_{I} G\right)^{\prime \prime}\right)
\end{aligned}
$$

is conjugate to

$$
\begin{aligned}
& \left(\pi^{0}\left(L_{I} H_{1}\right)^{\prime \prime} \vee\left(\pi^{0}\left(L_{I} H_{1}\right)^{\prime} \cap \pi^{0}\left(L_{I} H_{2}\right)^{\prime \prime}\right)\right) \otimes\left(\pi^{0}\left(L_{I} H_{2}\right)^{\prime} \cap \pi^{0}\left(L_{I} G\right)^{\prime \prime}\right) \subset \\
& \pi^{0}\left(L_{I} H_{2}\right)^{\prime \prime} \otimes\left(\pi^{0}\left(L_{I} H_{2}\right)^{\prime} \cap \pi^{0}\left(L_{I} G\right)^{\prime \prime}\right),
\end{aligned}
$$


hence it is irreducible and by Cor. 2.2 of [L6], its statistical dimension is $d\left(H_{2} / H_{1}\right)$.

By lemma 3.1, the minimal normal faithful conditional expectation $\epsilon_{1}$ restricts to a normal faithful conditional expectation $\eta$ from

$$
\pi^{0}\left(L_{I} H_{2}\right)^{\prime \prime} \vee\left(\pi^{0}\left(L_{I} H_{2}\right)^{\prime} \cap \pi^{0}\left(L_{I} G\right)^{\prime \prime}\right)
$$

to

$$
\pi^{0}\left(L_{I} H_{1}\right)^{\prime \prime} \vee\left(\pi^{0}\left(L_{I} H_{1}\right)^{\prime} \cap \pi^{0}\left(L_{I} H_{2}\right)^{\prime \prime}\right) \vee\left(\pi^{0}\left(L_{I} H_{2}\right)^{\prime} \cap \pi^{0}\left(L_{I} G\right)^{\prime \prime}\right) .
$$

By Prop. 4.3 of [L4], $\eta$ is also minimal, and so $d_{\eta}=d\left(H_{2} / H_{1}\right) \leq d_{\epsilon_{1}}=d\left(G / H_{1}\right)$ by the definition of statistical dimension (cf. 4.1).

To prove (2), note that $\eta \epsilon_{1}=\epsilon$ is a minimal conditional expextation by Cor. 2.2 of [L6], and $d_{\epsilon} \geq d_{\epsilon_{1}}$. so we have $d\left(H_{2} / H_{1}\right) d\left(G / H_{2}\right)=d_{\epsilon} \geq d_{\epsilon_{1}}=d\left(G / H_{1}\right)$, where we have used multiplicativity of statistical dimensions (cf. 4.1).

We consider some examples when Prop. 3.1 can be applied.

The conformal inclusion $S U(n)_{m} \times S U(m)_{n} \subset S U(n m)_{1}$ has been considered in [X2] and the decomposition is given in Th. 1 of $[\mathrm{ABI}]$. Let $H=S U(n)$ be the first factor in the above inclusion.

Lemma 3.2. $\pi^{0}\left(L_{I} S U(n m)\right)^{\prime \prime} \cap \pi^{0}\left(L_{I} S U(n)\right)^{\prime}=\pi^{0}\left(L_{I} S U(m)\right)^{\prime \prime}$. So the irreducible conformal precosheaf of coset $S U(n) \subset S U(n m)_{1}$ is the irreducible conformal precosheaf of $L S U(m)$ at level $n$.

Proof. From the definition we have:

$$
\pi^{0}\left(L_{I} S U(n m)\right)^{\prime \prime} \cap \pi^{0}\left(L_{I} S U(n)\right)^{\prime} \supset \pi^{0}\left(L_{I} S U(m)\right)^{\prime \prime}
$$

Since (cf. remark after Prop. 2.2) the action of modular group of

$$
\pi^{0}\left(L_{I} S U(n m)\right)^{\prime \prime} \cap \pi^{0}\left(L_{I} S U(n)\right)^{\prime}
$$

with respect to the vacuum vector $\Omega$ is geometric and fixes globally $\pi^{0}\left(L_{I} S U(m)\right)^{\prime \prime}$, by Takesaki's theorem (cf. [MT] or P. 495 of [W2]), we just have to show that

$$
\overline{\pi^{0}\left(L_{I} S U(n m)\right)^{\prime \prime} \cap \pi^{0}\left(L_{I} S U(n)\right)^{\prime} \Omega} \subset \overline{\pi^{0}\left(L_{I} S U(m)\right)^{\prime \prime} \Omega} .
$$

By the decomposition of $H^{0}$ with respect to $L S U(n) \times L S U(m)$ in Th. 1 of [ABI], $\Omega=\Omega_{0,0} \otimes \Omega_{0} \in H_{0,0} \otimes H_{0}$, where $H_{0,0}$ and $H_{0}$ are vacuum representations of $L S U(m)$ and $L S U(n)$ respectively, and $\Omega_{0,0}, \Omega_{0}$ are vacuum vectors for $L S U(m)$ and $L S U(n)$ respectively. By Reeh-Schlieder Theorem in Prop. 2.1, $\overline{\pi^{0}\left(L_{I} S U(m)\right)^{\prime \prime} \Omega}=H_{0,0} \otimes \Omega_{0}$, but by the observation before Prop. 2.2 we have

$$
\overline{\pi^{0}\left(L_{I} S U(n m)\right)^{\prime \prime} \cap \pi^{0}\left(L_{I} S U(n)\right)^{\prime} \Omega} \subset H_{0,0} \otimes \Omega_{0} .
$$


It follows that

$$
\overline{\pi^{0}\left(L_{I} S U(n m)\right)^{\prime \prime} \cap \pi^{0}\left(L_{I} S U(n)\right)^{\prime} \Omega} \subset \overline{\pi^{0}\left(L_{I} S U(m)\right)^{\prime \prime} \Omega},
$$

and the lemma is proved.

By lemma 3.2, Th. 1.2 of [X1] and formula (3.1), the inclusion $S U(n)_{k+l} \subset$ $S U(n(k+l))_{1}$ is cofinite. Since $S U(n)_{k+l} \subset S U(n)_{k} \times S U(n)_{l} \subset S U(n(k+l))_{1}$ where the first inclusion is diagonal, by (1) of Prop. 3.1 the diagonal inclusion $S U(n)_{k+l} \subset S U(n)_{k} \times S U(n)_{l}$ is cofinite, and use (2) of Prop. 3.1 repeatedly we conclude that the diagonal inclusion $S U(n)_{k} \subset S U(n)_{1} \times \ldots \times S U(n)_{1}$ is also cofinite, where there are $k$ factors in the product. It follows by (1) Prop. 3.1 that $S U(n)_{k_{1}+\ldots+k_{m}} \subset S U(n)_{k_{1}} \times \ldots \times S U(n)_{k_{m}}$ is cofinite, $k_{i} \in \mathbb{N}, i=1, \ldots, m$, since

$$
S U(n)_{k_{1}+\ldots+k_{m}} \subset S U(n)_{k_{1}} \times \ldots \times S U(n)_{k_{m}} \subset S U(n)_{1} \times \ldots S U(n)_{1}
$$

where there are $k_{1}+\ldots+k_{m}$ factors in the last group.

Suppose $H_{k} \subset G_{1}$ is a conformal inclusion, $H$ is simple and of type $A, G$ is simple and $k$ is the Dynkin index (cf. P. 170 of $[\mathrm{KW}]$ ). An infinite list can be found in [X2]. Let $l \in \mathbb{N}$. Since $H_{k l} \subset H_{k} \times \ldots \times H_{k}$ is cofinite by the previous paragraph and $H_{k} \times \ldots \times H_{k} \subset G_{1} \times \ldots \times G_{1}$ is cofinite by Prop. 2.4 of [X1], it follows by (2) of Prop. 3.1 that $H_{k l} \subset G_{1} \times \ldots \times G_{1}$ is cofinite, and by (1) of Prop. $3.1 H_{k l} \subset G_{l}$ is cofinite.

Finally let us consider the case $H \subset G_{m}$ with $G=S U(l)$ and $H$ is the Cartan subalgebra of $G$, a $l-1$ dimensional torus. We will first consider the inclusion

$$
H \subset G_{1} \times \ldots \times G_{1}
$$

where there are $m$ factors in the product, and the inclusion is diagonal. Define $\tilde{G}:=G \times G \ldots \times G$ where there are $m$ factors in the product.

The irreducible projective representations of $L H$ at level $m$ have been classified in Prop. 9.5.10 of [PS]. Let us describe this result in our case. These irreducible projective representations are in fact representations of $\mathcal{L} H$, which is a central extension of $L H$ induced from the central extension $\mathcal{L} G$ of $L G$ (cf. P. 483 of [W2] or Chap. 4 of $[\mathrm{PS}])$. Write $L H \simeq \operatorname{Hom}\left(S^{1}, H\right) \times H \times V$, where $H$ is the subgroup of constant loops, and $V$ is the vector space of maps $f: S^{1} \rightarrow \operatorname{Lie}(H)$ with integral 0 , which is regarded as a subgroup of $L H$ by the exponential map. The dentity component of $\mathcal{L} H$ is canonically a product $H \times \tilde{V}$, where $\tilde{V}$ is the Heisenberg group defined by a skew form on $V$. The center of the identity component of $\mathcal{L} H$ is $H \times S^{1}$. Let $\xi=\left(\xi_{1}, \ldots, \xi_{l-1},\left(\xi_{1} \ldots \xi_{l-1}\right)^{-1}\right) \in L H$, where each $\xi_{i} \in C^{\infty}\left(S^{1}, S^{1}\right)$ has winding number $x_{i}, i=1, \ldots, l-1$. The conjugate action of $\xi$ on the center $H \times S^{1}$ of the identity component of $\mathcal{L} H$ is given by (cf. P. 192 of [PS])

$$
(t, u) \rightarrow\left(t, u t_{1}^{x_{1}+\left(x_{1}+\ldots x_{l-1}\right)} \ldots t_{l-1}^{x_{l-1}+\left(x_{1}+\ldots x_{l-1}\right)}\right)
$$


where $t=\left(t_{1}, \ldots, t_{l-1},\left(t_{1} \ldots t_{l-1}\right)^{-1}\right) \in H$. Introduce an equivalent relation on $\mathbb{Z}^{l-1}$ by: $\left(n_{1}, \ldots, n_{l-1}\right) \sim\left(n_{1}^{\prime}, \ldots, n_{l-1}^{\prime}\right)$ iff there exists $\left(m_{1}, \ldots m_{l-1}\right) \in \mathbb{Z}^{l-1}$ with $m_{1}+$ $\ldots+m_{l-1} \in l \mathbb{Z}$ such that $\left(n_{1}^{\prime}, \ldots, n_{l-1}^{\prime}\right)=\left(n_{1}+m m_{1}, \ldots n_{l-1}+m m_{l-1}\right)$. Denote the equivalence class of $\left(n_{1}, \ldots, n_{l-1}\right)$ by $\left[n_{1}, \ldots, n_{l-1}\right]$ or simply $[n]$. The irreducible representation of $\mathcal{L} H$ at level $m$ on Hilbert space $H_{[n]}$ has the following form (cf. P. 192 of $[\mathrm{PS}])$ :

$$
H_{[n]}=\bigoplus_{\left(a_{1}, \ldots, a_{l-1}\right) \sim\left(n_{1}, \ldots, n_{l-1}\right)} H_{\left(a_{1}, \ldots, a_{l-1}\right)},
$$

where on $H_{\left(a_{1}, \ldots, a_{l-1}\right)}$, the center $H \times S^{1}$ of the identity componenet of $\mathcal{L} H$ acts as $(t, u) \rightarrow t_{1}^{a_{1}} \ldots t_{l-1}^{a_{l-1}} u \times \mathrm{id}$, and on $H_{\left(a_{1}, \ldots, a_{l-1}\right)}$ the representation of the Heisenberg group $\tilde{V}$ is irreducible (and unique by Prop. 9.5.10 of [PS]).

When restricting to $\mathcal{L} H$, the vacuum representation $\pi^{0}$ of $\mathcal{L} \tilde{G}:=\mathcal{L} G \times \ldots \mathcal{L} G$ (there are $\mathrm{m}$ factors in the product) on $\left(H_{v}\right)^{\otimes m}$ decomposes as:

$$
\left(H_{v}\right)^{\otimes m}=\sum_{l \mid \sum_{i} n_{i}} H_{0,[n]} \otimes H_{\left[n_{1}, \ldots n_{l-1}\right]},
$$

and $\pi_{[n]}$ are irreducible projective representations of $\mathcal{L} H$ on $H_{\left[n_{1}, \ldots n_{l-1}\right]}$ (cf. $\S 2.6$ of $[\mathrm{KW}])$. Let $\alpha \in L H \times \ldots \times L H$ be a loop of the form $\xi \times 1 \times \ldots \times 1$, where $\xi=\left(\xi_{1}, \ldots, \xi_{l-1},\left(\xi_{1} \ldots \xi_{l-1}\right)^{-1}\right) \in L H$, and each $\xi_{i} \in C^{\infty}\left(S^{1}, S^{1}\right)$ has winding number $x_{i}, i=1, \ldots, l-1$. We can assume that $\alpha$ is localized on $I$. Define

$$
A d_{\alpha} \cdot y:=\alpha y \alpha^{-1}, A d_{\alpha} \cdot \pi^{0}(y)=\pi^{0}(\alpha) \pi^{0}(y) \pi^{0}\left(\alpha^{-1}\right), \forall y \in \mathcal{L} \tilde{G} .
$$

Note (remember that $\mathcal{L} H$ is diagonally included in $\mathcal{L} \tilde{G}$ )

$$
A d_{\alpha} . y \in \mathcal{L} H, A d_{\alpha} \cdot \pi^{0}(y) \in \pi^{0}(\mathcal{L} H), \forall y \in \mathcal{L} H
$$

Then by definitions we have:

$$
\pi_{[n]}\left(A d_{\alpha} y\right) \simeq \pi_{[n+b]}(y), \forall y \in \mathcal{L} H
$$

where $[n+b]=\left[n_{1}+b_{1}, \ldots, n_{l-1}+b_{l-1}\right]$, with $b_{i}=x_{i}+\left(x_{1}+\ldots x_{l-1}\right), i=1, \ldots, l-1$. Note that this implies that $\pi_{[n]}$ has statistical dimension 1 since $A d_{\alpha}$ is a localized automorphism. Also note that since $A d_{\alpha}$ is an automorphism of $\pi^{0}(\mathcal{L} H)$, it is also an automorphism of $\pi^{0}(\mathcal{L} H)^{\prime} \cap \pi^{0}\left(\mathcal{L}{ }_{J} \tilde{G}\right)^{\prime \prime}$ for any interval $J$.

We claim that

$$
\pi_{0,[n]}\left(A d_{\alpha} \cdot y\right) \simeq \pi_{0,[n+b]}(y)
$$

for any $y \in A(J)$, where $A(J)$ is the conformal precosheaf for the coset $H \subset \tilde{G}$. In fact let $U_{[n]}: H_{[n]} \rightarrow H_{[n+b]}$ be a unitary map intertwinning the action of $\mathcal{L} H$ and the action of $A d_{\alpha} \cdot \mathcal{L} H$, and let $W=V \otimes U: H^{0} \rightarrow H^{0}$ be a unitary map such that $V \otimes U(z \otimes y)=V_{[n]} z \otimes U_{[n]} y \in H_{0,[n+b]} \otimes H_{[n+b]}$ for any $z \otimes y \in H_{0,[n]} \otimes H_{[n]}$. It follows that

$$
W^{*} \pi^{0}(\alpha) \in \pi^{0}(\mathcal{L} H)^{\prime}=\oplus_{[n]} B\left(H_{0,[n]}\right) \otimes i d_{H_{[n]}},
$$


so we have

$$
\pi^{0}(\alpha)=\oplus_{[n]} V_{[n]}^{\prime} \otimes U_{[n]}
$$

where $V_{[n]}^{\prime}: H_{0,[n]} \rightarrow H_{0,[n+b]}$ is unitary. Note $\pi^{0}\left(A d_{\alpha} . y\right)=\pi^{0}(\alpha) \pi^{0}(y) \pi^{0}(\alpha)^{-1}$, and

$$
\pi^{0}(y) \in \oplus_{[n]} B\left(H_{0,[n]}\right) \otimes i d_{H_{[n]}}, \forall y \in A(J)
$$

Hence

$$
\pi_{0,[n]}\left(A d_{\alpha} \cdot y\right)=V_{[n]}^{\prime} \pi_{0,[n+b]}(y) V_{[n]}^{\prime *}, \forall y \in A(J) .
$$

Now choose $x$ so that $b_{i}=-n_{i}, i=1, \ldots, l-1$, we get

$$
\pi_{0,[n]}\left(A d_{\alpha} \cdot y\right)=V_{[n]}^{\prime} \pi_{0,[0]}(y) V_{[n]}^{*} .
$$

So $\pi_{0,[n]}$ has the same statistical dimension as $\pi_{0,[0]}$ since $A d_{\alpha}$ is a localized automorphism.

If $\pi_{0,[0]}$ is the vacuum representation, then $\pi_{0,[n]}$ has statistical dimension 1 . Note $\pi_{[n]}$ also has statistical dimension 1 , by formula (3.1) we conclude that the diagonal inclusion $H \subset G_{1} \times \ldots \times G_{1}$ is cofinite.

We claim that $\pi_{0,[0]}$ is indeed the vacuum representation. Note this does not follow directly from Th. 2.3 since we assume $H$ is simply connected in the theorem. However, the assumption that $H$ is simply connected is only used in the proof of lemma 6. From the proof of lemma 6 , we see that the smeared vertex operators in Prop. 2.3 are affiliated with von Neumann algebra

$$
\pi^{0}\left((L H)^{0}\right)^{\prime} \cap \pi^{0}\left(L_{I} \tilde{G}\right)^{\prime \prime}
$$

where $(L H)^{0}$ is the connected component of $L H$ that contains identity. Note that $L H$ is generated as a group by $(L H)^{0}$ and a set of elements with non-trivial winding numbers, and we can certainly choose these elements to be in $L_{I^{\prime}} H \subset L_{I^{\prime}} \tilde{G}$. So

$$
\pi^{0}(L H)^{\prime \prime} \subset \pi^{0}\left((L H)^{0}\right)^{\prime \prime} \vee \pi^{0}\left(L_{I^{\prime}} \tilde{G}\right)^{\prime \prime}
$$

Hence if $p \in \pi^{0}\left((L H)^{0}\right)^{\prime} \cap \pi^{0}\left(L_{I} \tilde{G}\right)^{\prime \prime}$, then $p \in \pi^{0}(L H)^{\prime} \cap \pi^{0}\left(L_{I} \tilde{G}\right)^{\prime \prime}$. On the other hand

$$
\pi^{0}\left((L H)^{0}\right)^{\prime} \cap \pi^{0}\left(L_{I} \tilde{G}\right)^{\prime \prime} \supset \pi^{0}(L H)^{\prime} \cap \pi^{0}\left(L_{I} \tilde{G}\right)^{\prime \prime}
$$

So

$$
\pi^{0}\left((L H)^{0}\right)^{\prime} \cap \pi^{0}\left(L_{I} \tilde{G}\right)^{\prime \prime}=\pi^{0}(L H)^{\prime} \cap \pi^{0}\left(L_{I} \tilde{G}\right)^{\prime \prime} .
$$

This shows that Prop. 2.3, and therefore Th. 2.3 hold for any pair $H \subset \tilde{G}$ as long as $\tilde{G}$ is semisimple and simply connected. It follows now that Prop. 3.1 can be applied to the present case for $H \subset G_{m} \subset G_{1} \times \ldots \times G_{1}$ since we only use Th. 2.3 in its proof. So we conclude that $H \subset G_{m}$ is cofinite.

To summarize, we have proved the following: 
Corollary 3.1. The following inclusions are cofinite:

(1) $G_{k_{1}+k_{2}+\ldots+k_{m}} \subset G_{k_{1}} \times \ldots \times G_{k_{m}}$ where the inclusion is diagonal, $k_{i} \in \mathbb{N}, i=$ $1, \ldots, m$ and $G=S U(n)$;

(2) $H_{l k} \subset G_{l}$, if $H_{k} \subset G_{1}$ is a conformal inclusion where $k$ is the Dynkin index, $l \in \mathbb{N}, H$ is simple and of type $A$ and $G$ is simple;

(3) $H \subset G_{m}$, where $H$ is the Cartan subgroup of $G$.

\section{$\S 4$. BRAIDED ENDOMORPHISMS}

All the cosets considered in this section are assumed to verify the assumptions of Th. 2.3 unless stated otherwise.

4.1 Some results from $[\mathrm{X} 1]$. In this subsection we recall some of the results from [X1] which will be used in 4.2. We start with some preliminaries on sectors to set up notations.

Let $M$ be a properly infinite factor and $\operatorname{End}(M)$ the semigroup of unit preserving endomorphisms of $M$. In this paper $M$ will always be a type $I I I_{1}$ factor. Let $\operatorname{Sect}(M)$ denote the quotient of $\operatorname{End}(M)$ modulo unitary equivalence in $M$. It follows from [L3] and [L4] that $\operatorname{Sect}(M)$ is endowed with a natural involution $\theta \rightarrow \bar{\theta}$, and $\operatorname{Sect}(M)$ is a semiring: i.e., there are two operations,$+ \times$ on $\operatorname{Sect}(M)$ which verifes the usual axioms. The multiplication of sectors is simply the composition of sectors. Hence if $\theta_{1}, \theta_{2}$ are two sectors, we shall write $\theta_{1} \times \theta_{2}$ as $\theta_{1} \theta_{2}$. In [X1], the image of $\theta \in \operatorname{End}(M)$ in $\operatorname{Sect}(M)$ is denoted by $[\theta]$. However, since we will be mainly concerned with the ring structure of certain sectors in section 4 , we will denote $[\theta]$ simply by $\theta$ if no confusion arises.

Assume $\theta \in \operatorname{End}(M)$, and there exists a normal faithful conditional expectation $\epsilon: M \rightarrow \theta(M)$. We define a number $d_{\epsilon}$ (possibly $\infty$ ) by:

$$
d_{\epsilon}^{-2}:=\operatorname{Max}\left\{\lambda \in[0,+\infty) \mid \epsilon\left(m_{+}\right) \geq \lambda m_{+}, \forall m_{+} \in M_{+}\right\}
$$

(cf. $[\mathrm{PP}])$.

If $d_{\epsilon}<\infty$ for some $\epsilon$, we say $\theta$ has finite index or statistical dimension. In this case we define

$$
d_{\theta}=\operatorname{Min}_{\epsilon}\left\{d_{\epsilon} \mid d_{\epsilon}<\infty\right\} .
$$

$d_{\theta}$ is called the statistical dimension of $\theta . d_{\theta}^{2}$ is called the minimal index of $\theta$. In fact in this case there exists a unique $\epsilon_{\theta}$ such that $d_{\epsilon_{\theta}}=d_{\theta} \cdot \epsilon_{\theta}$ is called the minimal conditional expectation. It is clear from the definition that the statistical dimension of $\theta$ depends only on the unitary equivalence classes of $\theta$. When $N \subset M$ with $N \simeq M$, we choose $\theta \in \operatorname{End}(M)$ such that $\theta(M)=N$. The statistical dimension (resp. minimal index) of the inclusion $N \subset M$ is defined to be the statistical dimension (resp. minimal index) of $\theta$.

Let $\theta_{1}, \theta_{2} \in \operatorname{Sect}(M)$. By Th. 5.5 of [L3], $d_{\theta_{1}+\theta_{2}}=d_{\theta_{1}}+d_{\theta_{2}}$, and by Cor. 2.2 of [L6], $d_{\theta_{1} \theta_{2}}=d_{\theta_{1}} d_{\theta_{2}}$. These two properties are usually referred to as the additivity and multiplicativity of statistical dimensions. Also note by Prop. 4.12 of [L4] $d_{\theta}=d_{\bar{\theta}}$. If a sector does not have finite statistical dimension in any of the 
above three equations, then the equation is understood as the statement that both sides of the equation are $\infty$.

Assume $\lambda, \mu$, and $\nu \in \operatorname{End}(M)$ have finite statistical dimensions. Let $\operatorname{Hom}(\lambda, \mu)$ denote the space of intertwiners from $\lambda$ to $\mu$, i.e. $a \in \operatorname{Hom}(\lambda, \mu)$ iff $a \lambda(p)=\mu(p) a$ for any $p \in M \cdot \operatorname{Hom}(\lambda, \mu)$ is a finite dimensional vector space and we use $\langle\lambda, \mu\rangle$ to denote the dimension of this space. Note that $\langle\lambda, \mu\rangle$ depends only on $[\lambda]$ and $[\mu]$. Moreover we have $\langle\nu \lambda, \mu\rangle=\langle\lambda, \bar{\nu} \mu\rangle,\langle\nu \lambda, \mu\rangle=\langle\nu, \mu \bar{\lambda}\rangle$ which follows from Frobenius duality (See [L2] or [Y]). We will also use the following notation: if $\mu$ is a subsector of $\lambda$, we will write as $\mu \prec \lambda$ or $\lambda \succ \mu$. A sector is said to be irreducible if it has only one subsector.

Let $\theta_{i}, i=1, \ldots, n$ be a set of irreducible sectors with finite index. The ring generated by $\theta_{i}, i=1, \ldots, n$ under compositions is defined to be a vector space (possibly infinite dimensional) over $\mathbb{C}$ with a basis $\left\{\xi_{j}, j \geq 1\right\}$, such that $\xi_{j}$ are irreducible sectors, $\xi_{j} \neq \xi_{j^{\prime}}$ if $j \neq j^{\prime}$, and the set $\left\{\xi_{j}, j \geq 1\right\}$ is a list of all irreducible sectors which appear as subsectors of finite products of $\theta_{i}, i=1, \ldots, n$. The ring multiplication on the vector space is obtained naturally from that of $\operatorname{Sect}(M)$.

Let $M(J), J \in \mathcal{I}$ be an irreducible conformal precosheaf on Hilbert space $H^{0}$. Suppose $N(J), J \in \mathcal{I}$ is an irreducible conformal precosheaf and $\pi^{0}$ is a covariant representation of $N(J)$ on $H^{0}$ such that $\pi^{0}(N(J)) \subset M(J)$ is a directed standard net as defined in Definition 3.1 of [LR] for any directed set of intervals. Fix an interval $I$ and denote by $N:=N(I), M:=M(I)$. For any covariant representation $\pi_{\lambda}$ (resp. $\pi^{i}$ ) of the irreducible conformal precosheaf $N(J), J \in \mathcal{I}$ (resp. $M(J), J \in \mathcal{I}$ ), let $\lambda$ (resp. $i$ ) be the corresponding endomorphism of $N$ (resp. $M$ ) as defined in $\S 2.1$ of [GL]. These endomorphisms are obtained by localization in $\S 2.1$ of [GL] and will be referred to as localized endomorphisms for convenience. The corresponding sectors will be called localized sectors. See the paragraph after the proof of Lemma 4.2 for examples.

We will use $d_{\lambda}$ and $d_{i}$ to denote the statistical dimensions of $\lambda$ and $i$ respectively. $d_{\lambda}$ and $d_{i}$ are also called the statistical dimensions of $\pi_{\lambda}$ and $\pi^{i}$ respectively, and they are independent of the choice of $I$ (cf. Prop. 2.1 of [GL]).

Let $\pi^{i}$ be a covariant representation of $M(J), J \in \mathcal{I}$ which decomposes as:

$$
\pi^{i}=\sum_{\lambda} b_{i \lambda} \pi_{\lambda}
$$

when restricted to $N(J), J \in \mathcal{I}$, where the sum is finite and $b_{i \lambda} \in \mathbb{N}$. Let $\gamma_{i}:=$ $\sum_{\lambda} b_{i \lambda} \lambda$ be the corresponding sector of $N$. It is shown (cf. (1) of Prop. 2.8 in [X1]) that there are sectors $\rho, \sigma_{i} \in \operatorname{Sect}(N)$ such that:

$$
\rho \sigma_{i} \bar{\rho}=\gamma_{i}
$$

Notice that $\sigma_{i}$ are in one-to-one correspondence with covariant representations $\pi^{i}$, and in fact the map $i \rightarrow \sigma_{i}$ is an isomorphism of the ring generated by $i$ and the ring generated by $\sigma_{i}$. The subfactor $\bar{\rho}(N) \subset N$ is conjugate to $\pi^{0}(N(I)) \subset M(I)$ (cf. (2) of Prop. 2.6 in $[\mathrm{X} 1]$ ). 
Now we assume $\pi^{0}(N(I)) \subset M(I)$ has finite index. Then for each localized sector $\lambda$ of $N$ there exists a sector denoted by $a_{\lambda}$ of $N$ such that the following theorem is true (cf. $[\mathrm{X} 1])$ :

Theorem 4.1. (1) The map $\lambda \rightarrow a_{\lambda}$ is a ring homomorphism;

(2) $\rho a_{\lambda}=\lambda \rho, a_{\lambda} \bar{\rho}=\bar{\rho} \lambda, d_{\lambda}=d_{a_{\lambda}}$;

(3) $\left\langle\rho a_{\lambda}, \rho a_{\mu}\right\rangle=\left\langle a_{\lambda}, a_{\mu}\right\rangle=\left\langle a_{\lambda} \bar{\rho}, a_{\mu} \bar{\rho}\right\rangle$;

(4) $\left\langle\rho a_{\lambda}, \rho \sigma_{i}\right\rangle=\left\langle a_{\lambda}, \sigma_{i}\right\rangle=\left\langle a_{\lambda} \bar{\rho}, \sigma_{i} \bar{\rho}\right\rangle$;

(5) (3) (resp. (4)) remains valid if $a_{\lambda}, a_{\mu}$ (resp. $a_{\lambda}$ ) is replaced by any of its subsectors;

(6) $a_{\lambda} \sigma_{i}=\sigma_{i} a_{\lambda}$

Proof. (1) to (4) follows from Th. 3.1, 3.3, Cor. 3.2, lemma 3.4, 3.5 of [X1], (5) is proved on P. 9 of [X2], and (6) is proved on P. 387 of [X1]. It should be noted that these results in $\S 3$ of [X1] are stated for conformal inclusions, but all the proof there applies verbatim to the present setting.

4.2 The ring structure. We will apply the results of 4.1 to the case when $N(I)=$ $A(I) \otimes \pi_{0}\left(L_{I} H\right)^{\prime \prime}$ and $M(I)=\pi^{0}\left(L_{I} G\right)^{\prime \prime}$ under the assumption that $H \subset G_{L}$ is cofinite, where $A(I)$ is as in Prop. 2.2 for the coset $H \subset G_{L}$, and $\pi_{0}$ denotes the vacuum representation of $L H$. Note that if $H \subset G_{L}$ is cofinite, then $\pi^{0}(N(I)) \subset$ $M(I)$ has finite index. By Th. 4.1, for every localized endomorphisms $\lambda$ of $N(I)$ we have a map $a: \lambda \rightarrow a_{\lambda}$ which verifies (1) to (6) in Th. 4.1.

Tensor Notation. Let $\theta \in \operatorname{End}\left(A(I) \otimes \pi_{0}\left(L_{I} H\right)^{\prime \prime}\right)$. We will denote $\theta$ by $\rho_{1} \otimes \rho_{2}$ if

$$
\theta(p \otimes 1)=\rho_{1}(p) \otimes 1, \forall p \in A(I), \theta\left(1 \otimes p^{\prime}\right)=1 \otimes \rho_{2}\left(p^{\prime}\right), \forall p^{\prime} \in \pi_{0}\left(L_{I} H\right)^{\prime \prime},
$$

where $\rho_{1} \in \operatorname{End}(A(I)), \rho_{2} \in \operatorname{End}\left(\pi_{0}\left(L_{I} H\right)^{\prime \prime}\right)$.

Lemma 4.2. (1) If $\theta=\rho_{1} \otimes \rho_{2}$, and

$$
\left[\rho_{1}\right]=\sum_{i}\left[\rho_{1 i}\right],\left[\rho_{2}\right]=\sum_{j}\left[\rho_{2 j}\right]
$$

where all the summations are finite. Then:

$$
[\theta]=\sum_{i, j}\left[\rho_{1 i} \otimes \rho_{2 j}\right]
$$

$$
\left\langle\rho_{1} \otimes \rho_{2}, \sigma_{1} \otimes \sigma_{2}\right\rangle=\left\langle\rho_{1}, \rho_{2}\right\rangle\left\langle\sigma_{1}, \sigma_{2}\right\rangle,
$$

where $\rho_{1}, \sigma_{1}$ are in $\operatorname{End}(A(I))$, and $\rho_{2}, \sigma_{2}$ are in $\operatorname{End}\left(\pi_{0}\left(L_{I} H\right)^{\prime \prime}\right)$.

Proof. (1) follows immediately from the definitions. By (1), we just have to show (2) in the case that $\rho_{1}, \sigma_{1}, \rho_{2}, \sigma_{2}$ are irreducible sectors. It is obvious that if $\rho_{1} \simeq$ 
$\sigma_{1}, \rho_{2} \simeq \sigma_{2}$ as sectors, then $\rho_{1} \otimes \rho_{2} \simeq \sigma_{1} \otimes \sigma_{2}$ as sectors of $N(I)$. Now suppose $\rho_{1} \otimes \rho_{2} \simeq \sigma_{1} \otimes \sigma_{2}$ as sectors of $N(I)$. This means there exists a unitary $u \in N(I)$ such that:

$$
u \rho_{1}(p) \otimes \rho_{2}\left(p^{\prime}\right)=\sigma_{1}(p) \otimes \sigma_{2}\left(p^{\prime}\right) u
$$

for any $p \in A(I), p^{\prime} \in \pi_{0}\left(L_{I} H\right)^{\prime \prime}$. By the statement on P. 123 of [Stra], there exists normal conditional expectation $E: N(I) \rightarrow A(I) \otimes 1$ such that $E(u) \neq 0$. Applying $E$ to the above equation and setting $p^{\prime}=1$, we have:

$$
E(u) \rho_{1}(p)=\sigma_{1}(p) E(u)
$$

Since $\rho_{1}, \sigma_{1}$ are irreducible and $E(u) \neq 0$, it follows that $\rho_{1} \simeq \sigma_{1}$ as sectors. Similarly one can show that $\rho_{2} \simeq \sigma_{2}$ as sectors. This proves $(2)$.

Recall from $2.1 \pi_{i, \alpha}$ of $A(I)$ are obtained in the decompositions of $\pi^{i}$ of $L G$ with respect to subgroup $L H$, and we denote the set of such $(i, \alpha)$ by exp. For any $J \in \mathcal{I}$, let $U(J)$ be a unitary operator from $H_{i, \alpha}$ to $H_{0,0}$ such that:

$$
\pi_{i, \alpha}(p)=U(J)^{*} \pi_{0,0}(p) U(J), \forall p \in A(J) .
$$

Recall $I$ is a fixed interval. Identify $H_{i, \alpha}$ with $H_{0,0}$ by $U\left(I^{\prime}\right)$, we may choose a representation unitarily equivalent to $\pi_{i, \alpha}$, still denoted by $\pi_{i, \alpha}$ on $H_{0,0}$, with the property that $\pi_{i, \alpha}\left(p^{\prime}\right)=p^{\prime}, \forall p^{\prime} \in A\left(I^{\prime}\right)$. It follows that $\pi_{i, \alpha}(A(I))$ commutes with $A\left(I^{\prime}\right)$. By Haag duality in Prop. 2.1, $\pi_{i, \alpha}(p) \in A(I), \forall p \in A(I)$, and so $\pi_{i, \alpha} \mid A(I) \in \operatorname{End}(A(I))$. We will denote $\pi_{i, \alpha} \mid A(I)$ by $(i, \alpha)$. The corresponding sector in $\operatorname{Sect}(A(I))$ is also denoted by $(i, \alpha)$ when no confuison arises. Note that $(i, \alpha)$ is an irreducible sector if and only if $\pi_{i, \alpha}$ is an irreducible covariant representation, since the coset conformal precosheaf $A(J), \forall J \in \mathcal{I}$ is stronly additive by the remarks in 2.1 after Prop. 2.2. In fact suppose $(i, \alpha)$ is an irreducible sector. Let $p \in\left(\vee_{J \in \mathcal{I}} \pi_{i, \alpha}(A(J))\right)^{\prime}$. Then $p \in \pi_{i, \alpha}\left(A\left(I^{\prime}\right)\right)^{\prime}=A(I)$. It follows that $p \in \operatorname{Hom}((i, \alpha),(i, \alpha))=\mathbb{C}$ since $(i, \alpha)$ is irreduicble. On the other hand if $\pi_{i, \alpha}$ is irreducible, and $p \in \operatorname{Hom}((i, \alpha),(i, \alpha))$. Then $p \in A(I)$ and so $p \in\left(\pi_{i, \alpha}\left(A\left(I^{\prime}\right)\right) \vee \pi_{i, \alpha}(A(I))\right)^{\prime}$. But

$$
\pi_{i, \alpha}\left(A\left(I^{\prime}\right)\right) \vee \pi_{i, \alpha}(A(I))=\vee_{J \in \mathcal{I}} \pi_{i, \alpha}(A(J))
$$

by the strong additivity of the coset conformal precosheaf, so

$$
p \in\left(\vee_{J \in \mathcal{I}} \pi_{i, \alpha}(A(J))\right)^{\prime}=\mathbb{C}
$$

since $\pi_{i, \alpha}$ is irreducible. Similarly one can show that $(i, \alpha) \succ(j, \beta)$ if and only of $\pi_{j, \beta}$ appears as a direct summand of $\pi_{i, \alpha}$, and $(i, \alpha)$ is equal to $(j, \beta)$ as sectors if and only $\pi_{i, \alpha}$ is unitarily equivalent to $\pi_{j, \beta}$.

Given $(i, \alpha) \in \operatorname{End}(A(I))$ as above, we define $(i, \alpha) \otimes 1 \in \operatorname{End}(N(I))$ so that:

$$
(i, \alpha) \otimes 1\left(p \otimes p^{\prime}\right)=(i, \alpha)(p) \otimes p^{\prime}, \forall p \in A(I), p^{\prime} \in \pi_{0}\left(L_{I} H\right)^{\prime \prime} .
$$


It is easy to see that $(i, \alpha) \otimes 1$ corresponds to the covariant representation $\pi_{i, \alpha} \otimes \pi_{0}$ of $N(I)$. Note that this notation agrees with our tensor notation above. Also note that for any covariant representation $\pi_{x}$ of $A(I)$, we can define a localized sector $x \otimes 1$ of $N(I)$ in the same way as in the case when $\pi_{x}=\pi_{i, \alpha}$.

Each covariant representation $\pi^{i}$ of $L G$ gives rise to an endomorphism $\sigma_{i} \in$ $\operatorname{End}(N(I))$ and (cf. subsetion 4.1)

$$
\rho \sigma_{i} \bar{\rho}=\gamma_{i}=\sum_{\alpha}(i, \alpha) \otimes(\alpha)
$$

where the summation is over those $\alpha$ such that $(i, \alpha) \in \exp$. So by the properties of statistical dimensions (cf. 4.1) $d_{i} d_{\rho}^{2}=\sum_{\alpha} d_{(i, \alpha)} d_{\alpha}$. Note that this is in fact formula (3.1), with $d_{\rho}=d(G / H)$ by definition.

Proposition 4.2. Assume $H \subset G_{L}$ is cofinite. We have:

(1) Let $x, y$ be localized sectors of $A(I)$ with finite index. Then

$$
\langle x, y\rangle=\left\langle a_{x \otimes 1}, a_{y \otimes 1}\right\rangle
$$

(2) If $(i, \alpha) \in \exp$, then $a_{(i, \alpha) \otimes 1} \prec a_{1 \otimes \bar{\alpha}} \sigma_{i}$;

(3) Denote by $d_{(i, \alpha)}$ the statistical dimension of $(i, \alpha)$. Then $d_{(i, \alpha)} \leq d_{i} d_{\alpha}$, where $d_{i}\left(\right.$ resp. $\left.d_{\alpha}\right)$ is the statistical dimension of $i$ (resp. $\left.\alpha\right)$.

Proof. Ad (1): By the assumption and Th. 4.1, we have

$$
\begin{aligned}
\left\langle a_{x \otimes 1}, a_{y \otimes 1}\right\rangle & =\left\langle\rho a_{x \otimes 1}, \rho a_{y \otimes 1}\right\rangle \\
& =\langle(x \otimes 1) \rho,(y \otimes 1) \rho\rangle \\
& =\langle x \otimes 1,(y \otimes 1) \rho \bar{\rho}\rangle \\
& =\left\langle x \otimes 1,(y \otimes 1) \sum_{\delta}(0, \delta) \otimes \delta\right\rangle \\
& =\left\langle x \otimes 1, \sum_{\delta} y(0, \delta) \otimes \delta\right\rangle \\
& =\sum_{\delta}\langle x, y(0, \delta)\rangle \times\langle 1, \delta\rangle,
\end{aligned}
$$

where in the last identity we used (2) of lemma 4.2. Note $\langle 1, \delta\rangle$ is equal to 1 iff $\delta$ corresponds to the vacuum representation of $L H$. When $\delta$ is the vacuum representation, $(0, \delta)$ corresponds to the representation $\pi_{0,0}$ of $A(I)$, which by Th. 2.3 , is the vacuum representation of $A(I)$, and corresponds to the identity sector. So we have:

$$
\sum_{\delta}\langle x, y(0, \delta)\rangle \times\langle 1, \delta\rangle=\langle x, y\rangle
$$

and the proof of (1) is complete. 
Ad (2): Since $\pi^{i}$ has finite statistical dimension and $H \subset G$ is cofinite, by formula (3.1) $d_{(i, \alpha)}<\infty, d_{\alpha}<\infty, \forall(i, \alpha) \in \exp$. So we can assume $(i, \alpha)=\sum_{j} m_{j} x_{j}$, where the sum is finite, $m_{j} \in \mathbb{N}$, and $x_{j}$ is irreducible and has finite index. Note that $x_{j}$ is a localized sector of $A(I)$ (cf. Prop. 2.2 of [GL]), so $a_{x_{j} \otimes 1}$ is well defined, and it follows from (1) that $a_{x_{j} \otimes 1}$ is also irreducible. By Th. 4.1

$$
a_{(i, \alpha) \otimes 1}=\sum_{j} m_{j} a_{x_{j} \otimes 1} .
$$

By using Th. 4.1 and lemma 4.2 we have:

$$
\begin{aligned}
\left\langle a_{x_{i} \otimes 1}, a_{1 \otimes \bar{\alpha}} \sigma_{i}\right\rangle & =\left\langle a_{x_{i} \otimes 1} a_{1 \otimes \alpha}, \sigma_{i}\right\rangle \\
& =\left\langle a_{x_{i} \otimes \alpha}, \sigma_{i}\right\rangle \\
& =\left\langle a_{x_{i} \otimes \alpha}, \sigma_{i} \bar{\rho}\right\rangle \\
& =\left\langle\bar{\rho} x_{i} \otimes \alpha, \sigma_{i} \bar{\rho}\right\rangle \\
& =\left\langle x_{i} \otimes \alpha, \rho \sigma_{i} \bar{\rho}\right\rangle \\
& =\left\langle x_{i} \otimes \alpha, \sum_{\beta}(i, \beta) \otimes \beta\right\rangle \\
& =\left\langle x_{i},(i, \alpha)\right\rangle=m_{i} .
\end{aligned}
$$

This shows

$$
a_{(i, \alpha) \otimes 1}=\sum_{j} m_{j} a_{x_{j} \otimes 1} \prec a_{1 \otimes \bar{\alpha}} \sigma_{i} .
$$

(3) follows immediately from (2) and the fact that $(i, \alpha)$ and $a_{(i, \alpha) \otimes 1}$ have the same statistical dimension by (2) of Th. 4.1.

Theorem 4.2. Suppose $H \subset G_{L}$ is cofinite, and every irreducible representation $\pi_{\alpha}$ of $L H$ has finite index, and the localized sectors $\{\alpha\}$ generate a finite dimensional ring over $\mathbb{C}$ under compositions. Then Conj. 1 of 2.3 is true.

Proof. By (3) of Prop. 4.2, $d_{(i, \alpha)}<\infty, \forall(i, \alpha) \in \exp$, since $d_{i}<\infty, d_{\alpha}<\infty$ by our assumption. Hence each $(i, \alpha)$ decomposes into a direct sum of a finite number of irreducible sectors. By Prop. 2.2 of [GL], each $\pi_{i, \alpha}$ decomposes into a direct sum of a finite number of irreducible covariant representations.

By (6) of Th. 4.1, $a_{1 \otimes \bar{\alpha}} \sigma_{i}=\sigma_{i} a_{1 \otimes \bar{\alpha}}$, so the ring $Y$ generated by irreducible subsetors of $a_{1 \otimes \bar{\alpha}} \sigma_{i}, \forall \alpha, \forall i$ has finite dimension over $\mathbb{C}$ by (1) of Th. 4.1 and the the assumption of the theorem. Denote by $X$ the ring generated (under compositions) by the set of all irreducible sectors which appear as subsectors of $(i, \alpha), \forall(i, \alpha) \in \exp$. By Prop. 4.2, the map $x \in X \rightarrow a_{x \otimes 1}$ is an injective homomorphism from $X$ into $Y$. It follows that $X$ is finite dimensional over $\mathbb{C}$, and Conj. 1 of 2.3 is proved.

By Cor. 3.1, Th. 4.2 and the theorem on P. 535 of [W2], we immediately have the following: 
Corollary 4.2. Conj. 1 of 2.3 is true for the following inclusions:

(1) $G_{k_{1}+k_{2}+\ldots+k_{m}} \subset G_{k_{1}} \times \ldots \times G_{k_{m}}$ where the inclusion is diagonal , $k_{i} \in \mathbb{N}, i=$ $1, \ldots, m$ and $G=S U(n)$;

(2) $H_{l k} \subset G_{l}$, if $H_{k} \subset G_{1}$ is a conformal inclusion where $k$ is the Dynkin index $, l \in \mathbb{N}, l>1, H$ and $G$ are simple and of type $A$;

(3) $H \subset G_{m}$, where $H$ is the Cartan subgroup of $G, m \in \mathbb{N}, m>1$ and $G$ is a simple type A group.

Note in (2) and (3) of Cor. 4.2, we restrict $l>1$ and $m>1$ respectively to avoid the trivial case of conformal inclusions.

$4.3 S U(N) \subset S U(N)_{m^{\prime}} \times S U(N)_{m^{\prime \prime}}$. In this subsection we consider the coset $H \subset G_{L}$ with $H:=S U(N), G_{L}:=S U(N)_{m^{\prime}} \times S U(N)_{m^{\prime \prime}}$, where the embedding $H \subset G_{L}$ is diagonal. Let $\Lambda_{1}, \ldots, \Lambda_{N-1}$ be the fundamental weights of $\operatorname{sl}(N)$. Let $k \in \mathbb{N}$. Recall that the set of integrable weights of the affine algebra $\widehat{\operatorname{sl}(N)}$ at level $k$ is the following subset of the weight lattice of $\operatorname{sl}(N)$ :

$$
P_{++}^{(h)}=\left\{\lambda=\lambda_{1} \Lambda_{1}+\ldots+\lambda_{N-1} \Lambda_{N-1} \mid \lambda_{i} \in \mathbb{N}, \lambda_{1}+\ldots+\lambda_{N-1}<h\right\}
$$

where $h=k+N$. This set admits a $\mathbb{Z}_{N}$ automorphism generated by

$$
\sigma_{1}: \lambda=\left(\lambda_{1}, \lambda_{2}, \ldots, \lambda_{N-1}\right) \rightarrow \sigma_{1}(\lambda)=\left(h-\sum_{j=1}^{N-1} \lambda_{j}, \lambda_{1}, \ldots, \lambda_{N-2}\right) .
$$

We define the color $\tau(\lambda): \equiv \sum_{i}\left(\lambda_{i}-1\right) i \bmod (N)$ and $Q$ to be the root lattice of $\widehat{s l(N)}$ (cf. $\oint 1.3$ of $[\mathrm{KW}]$ ). Note that $\lambda \in Q$ if and only if $\tau(\lambda) \equiv 0 \bmod (N)$.

As in 2.1, we use $i$ (resp. $\alpha$ ) to denote the irreducible positive energy representations of $L G$ (resp. $L H)$. To compare our notations with that of $\S 2.7$ in $[\mathrm{KW}]$, note that our $i$ is $\left(\Lambda^{\prime}, \Lambda^{\prime \prime}\right)$ of $[\mathrm{KW}]$, and our $\alpha$ is $\Lambda$ of $\left[\mathrm{KW}\right.$. We will identify $i=\left(\Lambda^{\prime}, \Lambda^{\prime \prime}\right)$ and $\alpha=\Lambda$ where $\Lambda^{\prime}, \Lambda^{\prime \prime}, \Lambda$ are the weights of $s l(N)$ at levels $m^{\prime}, m^{\prime \prime}, m^{\prime}+m^{\prime \prime}$ respectively. Denote by $0^{\prime}, 0^{\prime \prime}, 0$ the vacuum representations of $\widehat{s l(N)}$ at level $m^{\prime}, m^{\prime \prime}$ and $m^{\prime}+m^{\prime \prime}$ respectively. Note that for $i=\left(\Lambda^{\prime}, \Lambda^{\prime \prime}\right), \alpha=\Lambda$, by Th. 1.2 of [X1] the statistical dimensions of $i$ and $\alpha$ are given by

$$
d_{i}=\frac{a\left(\Lambda^{\prime}\right) a\left(\Lambda^{\prime \prime}\right)}{a\left(0^{\prime}\right) a\left(0^{\prime \prime}\right)}, d_{\alpha}=\frac{a(\Lambda)}{a(0)}
$$

where the positive numbers $a(\Lambda), a\left(\Lambda^{\prime}\right)$ and $a\left(\Lambda^{\prime \prime}\right)$ are defined as in (0.4b) of $[\mathrm{KW}]$ $\left(a(\Lambda)\right.$ is also equal to $S_{\Lambda_{0}}^{(\Lambda)}$ as defined on P. 362 of $\left.[\mathrm{X} 1]\right)$.

Suppose

$$
i=\left(\Lambda_{1}{ }^{\prime}, \Lambda_{1}{ }^{\prime \prime}\right), j=\left(\Lambda_{2}{ }^{\prime}, \Lambda_{2}{ }^{\prime \prime}\right), k=\left(\Lambda_{3}{ }^{\prime}, \Lambda_{3}{ }^{\prime \prime}\right), \alpha=\Lambda_{1}, \beta=\Lambda_{2}, \delta=\Lambda_{3} .
$$

Then the fusion coefficients $N_{i j}^{k}:=N_{\Lambda_{1}{ }^{\prime} \Lambda_{2}{ }^{\prime}}^{\Lambda^{\prime}} N_{\Lambda_{1}{ }^{\prime \prime} \Lambda_{2}{ }^{\prime \prime}}^{\Lambda^{\prime \prime}}\left(\operatorname{resp} . \quad N_{\alpha \beta}^{\delta}:=N_{\Lambda_{1} \Lambda_{2}}^{\Lambda_{3}}\right.$ )of $L G$ (resp. $L H$ ) are well known and they are given by Verlinde formula (cf. Cor. 1 on P. 536 of [W2] and P. 288 of [Kac]). 
Recall $\pi_{i, \alpha}$ are the covariant representations of the coset $H \subset G_{L}$. The set of all $(i, \alpha):=\left(\Lambda^{\prime}, \Lambda^{\prime \prime}, \Lambda\right)$ which appear in the decompositions of $\pi^{i}$ of $L G$ with respect to $L H$ is denoted by exp. This set is determined on P. 194 of [KW] to be $\left(\Lambda^{\prime}, \Lambda^{\prime \prime}, \Lambda\right) \in \exp$ iff $\Lambda^{\prime}+\Lambda^{\prime \prime}-\Lambda \in Q$. The $\mathbb{Z}_{N}$ action on $(i, \alpha), \forall i, \forall \alpha$ is denoted by

$$
\sigma(i, \alpha):=(\sigma(i), \sigma(\alpha))=\left(\sigma\left(\Lambda^{\prime}\right), \sigma\left(\Lambda^{\prime \prime}\right), \sigma(\Lambda)\right), \sigma \in \mathbb{Z}_{N}
$$

This is also known as diagram automorphisms since they corresponds to the automorphisms of Dynkin diagrams. Note that $d_{\sigma(i)}=d_{i}, d_{\sigma(\alpha)}=d_{\alpha}$ by (3.2) of [Wal] and the formula for statistical dimensions above. Also note that this $\mathbb{Z}_{N}$ action preserves exp and therefore induces a $\mathbb{Z}_{N}$ action on exp. For each $(i, \alpha) \in \exp$, we will denote by $[i, \alpha]$ its orbit in exp under the $\mathbb{Z}_{N}$ action.

Theorem 4.3. Let $H \subset G_{L}$ be as in the previous paragraph. Then:

(1) $a_{(i, \alpha) \otimes 1}=\sigma_{i} a_{1 \otimes \bar{\alpha}}$ for all $(i, \alpha) \in \exp$;

(2) Assume the action of $Z_{N}$ on exp is faithful, i.e., if $\sigma(i)=i, \sigma(\alpha)=\alpha$ for some $(i, \alpha) \in \exp$, then $\sigma=i d$. Then the covariant representations $\pi_{i, \alpha}$ are irreducible and $\pi_{i, \alpha}$ is unitarily equivalent to $\pi_{j, \beta}$ as covariant representations iff $\sigma(i)=j, \sigma(\alpha)=\beta$ for some $\sigma \in \mathbb{Z}_{N}$;

(3) Suppose the conditions of (2) hold. Denote by EXP the set of all irreducible localized sectors corresponding to the the covariant representations $\pi_{i, \alpha}, \forall(i, \alpha) \in$ exp. Then the set EXP is in one to one correspondence with the set $\{[i, \alpha], \forall(i, \alpha) \in$ exp $\}$. Denote the elements of EXP by $[i, \alpha]$. Define

$$
C_{[i, \alpha][j, \beta]}^{[k, \delta]}:=\sum_{\sigma \in \mathbb{Z}_{N}} N_{i j}^{\sigma(k)} N_{\alpha \beta}^{\sigma(\delta)} .
$$

Then the compositions are given by:

$$
[i, \alpha][j, \beta]=\sum_{[k, \delta]} C_{[i, \alpha][j, \beta]}^{[k, \delta]}[k, \delta] ;
$$

(4) Conj. 2 of 2.3 is true for $H \subset G_{L}$.

Proof. By Cor. 4.2 and Th. 4.1, for any $(i, \alpha),\left(i^{\prime}, \alpha^{\prime}\right)$ we have:

$$
\begin{aligned}
\left\langle a_{1 \otimes \bar{\alpha}} \sigma_{i}, a_{1 \otimes \overline{\alpha^{\prime}}} \sigma_{i^{\prime}}\right\rangle & =\left\langle a_{1 \otimes \bar{\alpha}} a_{1 \otimes \alpha^{\prime}}, \sigma_{\bar{i}} \sigma_{i^{\prime}}\right\rangle \\
& =\left\langle a_{1 \otimes \bar{\alpha} \alpha^{\prime}}, \sigma_{\bar{i}}^{-} \sigma_{i^{\prime}}\right\rangle \\
& =\left\langle\sum_{\beta} N_{\bar{\alpha} \alpha^{\prime}}^{\beta} a_{1 \otimes \beta}, \sum_{j} N_{\bar{i} i^{\prime}}^{j} \sigma_{j}\right\rangle \\
& =\left\langle\sum_{\beta} N_{\bar{\alpha} \alpha^{\prime}}^{\beta} 1 \otimes \beta, \sum_{j, \delta} N_{\bar{i} i^{\prime}}^{j}(j, \delta) \otimes \delta\right\rangle \\
& =\sum_{\beta} N_{\bar{\alpha} \alpha^{\prime}}^{\beta} \sum_{j} N_{\bar{i} i^{\prime}}^{j}\langle 1,(j, \beta)\rangle,
\end{aligned}
$$


where 1 in the last $=$ stands for the identity sector of $A(I)$, which by Th. 2.3, corresponds to the representation $\pi_{0,0}$. So

$$
\langle 1,(j, \beta)\rangle \neq 0
$$

if and only if $\pi_{0,0}$ appears as an irreducible summand in $\pi_{j, \beta}$ by the remarks after lemma 4.2. Note that the vacuum vector (unique up to a nonzero scalar) of $\pi_{0,0}$ has lowest energy (the eigenvalue of the generator of the rotation group) 0 , and $\pi_{0,0}$ is the unique (up to unitary equivalence) irreducible representation with this property (cf. remarks after Prop. 2.1). So $\pi_{0,0}$ appears as an irreducible summand in $\pi_{j, \beta}$ if and only if there exists a nonzero (vacuum) vector in $H_{j, \beta}$ with lowest energy 0 . The set of such $(j, \beta)$ was introduced on P. 186 in $[\mathrm{KW}]$ with our $(j, \beta)$ corresponds to $(M, \mu \bmod (\delta))$ in the notation of $[\mathrm{KW}$ ] (in the notation on P. 186 of $[\mathrm{KW}], h_{M}-h_{\mu} \geq 0$ is the eigenvalue of the generator of the rotation group in the coset Hilbert space by definition). This set in our case of diagonal inclusions is determined in (2.7.12) of $[\mathrm{KW}$. Translate (2.7.12) of [KW] into the notations of this paper, the statement is that $\pi_{0,0}$ appears as an irreducible summand in $\pi_{j, \beta}$ iff there exists $\sigma \in \mathbb{Z}_{N}$ such that $\sigma(0,0)=(j, \beta)$, where 0 is used to denote the vacuum representation of $G$ and $H$. Since (cf. (3.3) of [Wal])

$$
N_{\bar{i} i^{\prime}}^{\sigma(0)}=\delta_{\sigma(i), i^{\prime}}, N_{\bar{\alpha} \alpha^{\prime}}^{\sigma(0)}=\delta_{\sigma(\alpha), \alpha^{\prime}},
$$

we have:

$$
\left\langle a_{1 \otimes \bar{\alpha}} \sigma_{i}, a_{1 \otimes \overline{\alpha^{\prime}}} \sigma_{i^{\prime}}\right\rangle=\sum_{\sigma \in \mathbb{Z}_{N}} \delta_{\sigma(i), i^{\prime}} \delta_{\sigma(\alpha), \alpha^{\prime}}\langle\sigma(0,0),(0,0)\rangle .
$$

Ad (1): We will prove (1) by using an "exhaustion" trick similar to the one used in $\S 3$ of $[\mathrm{X} 3]$.

Suppose $(i, \alpha)=\left(i^{\prime}, \alpha^{\prime}\right)=(0, \alpha)$, where 0 denotes the vacuum sector of $L G$. Note that $\sigma(0)=0$ iff $\sigma=1$. So we conclude from $(* *)$ that $a_{1 \otimes \bar{\alpha}}$ is irreducible. By $(2)$ of Prop. $4.2, a_{1 \otimes \bar{\alpha}}=a_{(0, \alpha) \otimes 1}$ if $(0, \alpha) \in \exp$, and so $d_{(0, \alpha)}=d_{\alpha}$.

Note that (cf. the paragraph before Prop. 4.2) for fixed $i$, the statistical dimension of $\rho \sigma_{i} \bar{\rho}$ is given by $\sum_{\alpha} d_{i, \alpha} d_{\alpha}$ where the sum is over those $\alpha$ with $(i, \alpha) \in \exp$, which will be denoted by $\exp _{i}$. Note that if $i=\left(\Lambda^{\prime}, \Lambda^{\prime \prime}\right), \alpha=\Lambda$, then $\exp _{i}$ is a congruence class of $P_{++}^{m^{\prime}+m^{\prime \prime}+N} \bmod Q$ (congruent to $\left.\Lambda^{\prime}+\Lambda^{\prime \prime}\right)$ by definition. So $d_{\rho}^{2}=\frac{1}{d_{i}} \sum_{\alpha \in \exp _{i}} d_{i, \alpha} d_{\alpha}$. By (3) of Prop. 4.2, $d_{(i, \alpha)} \leq d_{i} d_{\alpha}, \forall(i, \alpha) \in \exp$, hence

$$
d_{\rho}^{2} \leq \sum_{\alpha \in e x p_{i}} d_{\alpha} d_{\alpha}=\sum_{\alpha \in e x p_{0}} d_{\alpha} d_{\alpha}
$$

where tha last $=$ follows from Cor. 2.7 of $[\mathrm{KW}]^{3}$. But since $d_{(0, \alpha)}=d_{\alpha}$, we have:

$$
d_{\rho}^{2}=\sum_{\alpha \in \exp p_{0}} d_{\alpha} d_{\alpha}
$$

\footnotetext{
${ }^{3}$ Note that our $\alpha$ correspond to $\Lambda$ in Cor. 2.7 of $[\mathrm{KW}], d_{\alpha}=\frac{a(\Lambda)}{a(0)}$ where 0 denotes the vacuum representation, and $\exp _{i}$ is a congruence class of $P_{++}^{m^{\prime}+m^{\prime \prime}+N} \bmod Q$.
} 
It follows that all the $\leq$ 's above are actually $=$, in particular $d_{i, \alpha}=d_{i} d_{\alpha}$, and it follows from (2) of Prop. 4.2 that $a_{(i, \alpha) \otimes 1}=\sigma_{i} a_{1 \otimes \bar{\alpha}}$.

So we have (see the paragraph before Th. 4.3):

$$
d_{\sigma(0,0)}=d_{\sigma(0)} d_{\sigma(0)}=1
$$

On the otherhand

$$
\langle\sigma(0,0),(0,0)\rangle \geq 1
$$

it follows by comparing statistical dimensions that

$$
\langle\sigma(0,0),(0,0)\rangle=1
$$

So we can improve $(* *)$ to

$$
\left\langle a_{1 \otimes \bar{\alpha}} \sigma_{i}, a_{1 \otimes \overline{\alpha^{\prime}}} \sigma_{i^{\prime}}\right\rangle=\sum_{\sigma \in \mathbb{Z}_{N}} \delta_{\sigma(i), i^{\prime}} \delta_{\sigma(\alpha), \alpha^{\prime}}
$$

Ad (2): By assumption expression $(*)$ holds, for a unique $\sigma$ since the action is faithful. If $(i, \alpha)=\left(i^{\prime}, \alpha^{\prime}\right)$, then $\sigma(i)=i^{\prime}, \sigma(\alpha)=\alpha^{\prime}$ iff $\sigma=i d$, and we conclude from $(*)$ that

$$
\left\langle a_{1 \otimes \bar{\alpha}} \sigma_{i}, a_{1 \otimes \bar{\alpha}} \sigma_{i}\right\rangle=1,
$$

i.e., $a_{1 \otimes \bar{\alpha}} \sigma_{i}$ is irreducible. By (1) of Prop. 4.2 and (1) of Th. 4.3 we conclude that if $(i, \alpha) \in \exp$, then $(i, \alpha)$ is irreducible, i.e., $\pi_{i, \alpha}$ is irreducible by the remarks after lemma 4.2 .

If $\sigma(i)=j, \sigma(\alpha)=\beta$, then

$$
1 \geq\left\langle a_{1 \otimes \bar{\alpha}} \sigma_{i}, a_{1 \otimes \bar{\beta}} \sigma_{j}\right\rangle \geq 1
$$

where the first $\geq$ follows from the fact that $a_{1 \otimes \bar{\alpha}} \sigma_{i}, a_{1 \otimes \bar{\beta}} \sigma_{j}$ are irreducible, and the second $\geq$ follows from $(*)$. So we must have

$$
a_{1 \otimes \bar{\alpha}} \sigma_{i}=a_{1 \otimes \bar{\beta}} \sigma_{j}
$$

By (1) of Prop. 4.2 and (1) of Th. $4.3\langle(i, \alpha),(j, \beta)\rangle=1$, so $(i, \alpha)$ is identical to $(j, \beta)$ as sectors since both sectors are irreducible. Hence $\pi_{i, \alpha}$ is unitarily equivalent to $\pi_{j, \beta}$ as covariant representations by the remarks after lemma 4.2 . On the other hand if $\pi_{i, \alpha}$ is unitarily equivalent to $\pi_{j, \beta}$ as covariant representations, then $(i, \alpha)$ is equal to $(j, \beta)$ as sectors by the remarks after lemma 4.2. By (1) of Prop. 4.2 and (1) of Th. 4.3

$$
a_{1 \otimes \bar{\alpha}} \sigma_{i}=a_{1 \otimes \bar{\beta}} \sigma_{j}
$$

so we must have $\sigma(i)=j, \sigma(\alpha)=\beta$ for some $\sigma \in \mathbb{Z}_{N}$ by $\left.{ }^{*}\right)$. This proves (2). 
Ad (3): Note that if $(i, \alpha) \in \exp ,(j, \beta) \in \exp$, by (1) and Th. 4.1 we have:

$$
\begin{aligned}
a_{(i, \alpha) \otimes 1} a_{(j, \beta) \otimes 1} & =\sigma_{i} \sigma_{j} a_{1 \otimes \bar{\alpha}} a_{1 \otimes \bar{\beta}} \\
& =\sum_{k, \delta} N_{i j}^{k} N_{\alpha \beta}^{\delta} \sigma_{k} a_{1 \otimes \bar{\delta}}
\end{aligned}
$$

Note by the characterization of $\exp$ (cf. P. 194 of $[\mathrm{KW}]$ ) and 4.3 .4 of [FKW], if $N_{i j}^{k} N_{\alpha \beta}^{\delta} \neq 0$, then $(k, \delta) \in \exp$. Using (1) again we have:

$$
a_{(i, \alpha) \otimes 1} a_{(j, \beta) \otimes 1}=\sum_{(k, \delta) \in \exp } N_{i j}^{k} N_{\alpha \beta}^{\delta} a_{(k, \delta) \otimes 1}
$$

(3) now follows from (1) of Prop. 4.2 and (2).

Ad (4): By (1) we need to show that

$$
\frac{b(i, \alpha)}{b(0,0)}=d_{i} d_{\alpha}
$$

where under the identification of $i$ with $\left(\Lambda^{\prime}, \Lambda^{\prime \prime}\right)$ and $\alpha$ with $\Lambda, b(i, \alpha)$ is given by (2.7.14) of $[\mathrm{KW}]$, and use the notations there we have

$$
b(i, \alpha)=b\left(\Lambda^{\prime}, \Lambda^{\prime \prime} ; \Lambda\right)=N a\left(\Lambda^{\prime}\right) a\left(\Lambda^{\prime \prime}\right) a(\Lambda) .
$$

Note that $d(\Lambda)=\frac{a(\Lambda)}{a(0)}$, and we have similar identities when $\Lambda, 0$ is replaced by $\Lambda^{\prime}, 0^{\prime}$ and $\Lambda^{\prime \prime}, 0^{\prime \prime}$. The proof of (4) is now complete by definitions.

Note that by (1) of Th. 4.3 and formula $(*)$ if $\sigma(i)=i, \sigma(\alpha)=\alpha$ for some $\sigma \neq i d$, $(i, \alpha) \in \exp$, then $a_{(i, \alpha) \otimes 1}$ is not irreducible. Hence $(i, \alpha)$ is not irreducible by $(1)$ of Prop. 4.2. This happens for an example when $N=2, m^{\prime} \in 2 \mathbb{N}$ and $m^{\prime \prime}=2$, which is related to supersymmetric conformal algebras (cf. P. 195 of $[\mathrm{KW}]$ ).

Recall from subsection 2.3 coset $W_{N}$ algebras with critical parameters are defined to be the irreducible conformal precosheaves of cosets

$$
S U(N)_{m+1} \subset S U(N)_{m} \times S U(N)_{1}
$$

which obviously satisfies (1) to (4) of Th. 4.3. For $N=2$, Th. 4.3 is obtained in [Luke] by different methods. So we obtain a proof of a long-standing conjecture about representations of coset $W_{N}(N>2)$ algebras with critical parameters, which is similar in the type $A$ case to the conjecture 3.4 and Th. 4.3 stated in $\S 3$ and 4 of $\left[\mathrm{FKW}\right.$. To compare (3) of Th. 4.3 to Th. 4.3 of $[\mathrm{FKW}]$, note that $\left(p, p^{\prime}\right)$ in [FKW] is identified with $\left(m^{\prime}, m^{\prime}+1\right)$ in our case, and $\left(\lambda_{i}, \lambda_{i}^{\prime}\right)$ in Th. 4.3 of [FKW] is identified with $\left[\Lambda_{i}^{\prime}, \Lambda_{i}{ }^{\prime \prime}, \Lambda_{i}\right]$ in our case with $\lambda_{i}=\Lambda_{i}^{\prime}, \lambda_{i}^{\prime}=\Lambda_{i}$, and $\Lambda_{i}^{\prime \prime}$ is the unique element such that $\Lambda_{i}^{\prime}+\Lambda_{i}^{\prime \prime}-\Lambda_{i} \in Q$. Then the compostions in Th. 4.3 of [FKW] can be easily checked to be the same as (3) of Th. 4.3 under the assumptions of Th. 4.3 in $[\mathrm{FKW}]$. 
4.4 More examples. Let us consider the case $H \subset G_{m}$ with $G=S U(l)$ and $H$ is the Cartan subalgebra of $G$, a $l-1$ dimensional torus. This coset does not verify the conditions of Th. 2.3, but Th. 2.3 applies to this coset by the remark before Cor. 3.1. So we can apply the results of section 4 .

Recall the equivalent relation on $\mathbb{Z}^{l-1}$ in 3.1 defined by:

$$
\left(n_{1}, \ldots, n_{l-1}\right) \sim\left(n_{1}^{\prime}, \ldots, n_{l-1}^{\prime}\right)
$$

iff there exists $\left(m_{1}, \ldots m_{l-1}\right) \in \mathbb{Z}^{l-1}$ with $m_{1}+\ldots+m_{l-1} \in l \mathbb{Z}$ such that

$$
\left(n_{1}^{\prime}, \ldots, n_{l-1}^{\prime}\right)=\left(n_{1}+m m_{1}, \ldots n_{l-1}+m m_{l-1}\right) .
$$

Denote the equivalence class of $\left(n_{1}, \ldots, n_{l-1}\right)$ by $[n]:=\left[n_{1}, \ldots, n_{l-1}\right]$ or simply $[n]$, and they are used to denote irreducible projective representations of $L H$.

Let $\Lambda$ be an irreducible weight of $S U(l)$ at level $m$. Let $\tau(\Lambda)$ be the color of $\Lambda$, see 4.3. Then:

$$
H^{\Lambda}=\oplus_{\left\{[n] \mid\left(\sum_{i} n_{i}-\tau(\Lambda)\right) \in l \mathbb{Z}\right\}} H_{\Lambda,[n]} \otimes H_{[n]},
$$

cf. $\S 2.6$ of $[\mathrm{KW}]$. So the set $\exp =\left\{(\Lambda,[n]), \mid\left(\sum_{i} n_{i}-\tau(\Lambda)\right) \in l \mathbb{Z}\right\}$ is determined, and $(i, \alpha)=(\Lambda,[n])$ in the present case. Note that the sector $[n]$ corresponds to an automorphism and has statistical dimension 1 (cf. section 3 before Cor. 3.1). So $a_{1 \otimes[n]}$ has statistical dimension 1 , and it follows that $\overline{a_{1 \otimes[n]}} a_{1 \otimes[n]}$ is the identity sector. So

$$
\left\langle a_{1 \otimes[n]} \sigma_{\Lambda}, a_{1 \otimes[n]} \sigma_{\Lambda}\right\rangle=\left\langle\overline{a_{1 \otimes[n]}} a_{1 \otimes[n]} \sigma_{\Lambda}, \sigma_{\Lambda}\right\rangle=\left\langle\sigma_{\Lambda}, \sigma_{\Lambda}\right\rangle=1 .
$$

Hence $a_{1 \otimes[n]} \sigma_{\Lambda}$ is irreducible for any $[n], \Lambda$. So by Cor. 3.1 and (2) of Prop. 4.2 if $(\Lambda,[n]) \in \exp$,

$$
a_{(\Lambda,[n]) \otimes 1}=\overline{a_{1 \otimes[n]}} \sigma_{\Lambda},
$$

and by (1) of Prop. 4.2 we have the following fusion rules:

$$
(\Lambda,[n])\left(\Lambda^{\prime},\left[n^{\prime}\right]\right)=\sum_{\Lambda^{\prime \prime}} N_{\Lambda \Lambda^{\prime}}^{\Lambda^{\prime \prime}}\left(\Lambda^{\prime \prime},\left[n+n^{\prime}\right]\right),
$$

for any $(\Lambda,[n]),\left(\Lambda^{\prime},\left[n^{\prime}\right]\right) \in \exp$. By using (2.6.14) of $[\mathrm{KW}]$, one can immediately check that Conj. 2 of 2.3 is true in this case, as we did in the proof of (4) of Th. 4.3. Note this coset is related to Parafermions, see [DL] for an approach using vertex operator algebras.

Next we consider the case $S U(2)_{4 k} \subset S U(3)_{k}$. When $k=1, S U(2)_{4} \subset S U(3)_{1}$ is a conformal inclusion, so by Cor. 4.2 , the coset $S U(2)_{4 k} \subset S U(3)_{k}, k>1$ is rational, i.e., it verifies Conj. 1 of 2.3. In this particular example, we will use the notations in $\S 6$ of [DJ1]. Thus the weights of $\widehat{s l(2)}$ are labeled by an integer $l$ with 0 denoting the vacuum representation and the weights of $\widehat{s l(3)}$ are labeled by a pair of integers $(p q)$ with (00) denoting the vacuum representation. So $(i, \alpha):=(p q, l)$. 
In general, the identifications of $(i, \alpha)=(j, \beta)$ as sectors are related to certain Dynkin diagram automorphisms, as in (2) of Th. 4.3. See [SY] for more examples. However, in [DJ1], a "Maverick" coset is given which violates the above identification rule. This is the coset $S U(2)_{8} \subset S U(3)_{2}$. From the first line of table on P. 4117 of [DJ1], we have:

$$
(00,0)=(00,8)=(11,4)
$$

as sectors by Th. 2.3 and the fact that the vacuum representation is the unique (up to unitary equivalence) representation which contains a nonzero (vacuum) vector with lowest energy 0. By using the above equation, Th. 4.1 and Prop. 4.2 we find that the irreducible subsectors of $a_{(p q, l) \otimes 1}$ generate a 6 dimensional ring whose basis is:

$$
\begin{aligned}
1, x & :=a_{(00,4) \otimes 1}=a_{1 \otimes 4}-\sigma_{11}, \\
y & :=a_{(10,2)}=\sigma_{10} a_{1 \otimes 2}-\sigma_{02} a_{1 \otimes 2}, \\
\bar{y} & :=a_{(01,2)}=\overline{a_{(10,2)}} \\
z & :=a_{(10,4)}=\sigma_{02}, \bar{z}:=a_{(01,4)}=\overline{a_{(10,4)}}
\end{aligned}
$$

where $\bar{a}$ is the conjugate of $a$. The statistical dimensions of $a_{(00,4) \otimes 1}, a_{(10,2)}, a_{(10,4)}$ are given by $\frac{\sqrt{5}+1}{2}, \frac{\sqrt{5}+1}{2}, 1$ respectively. The fusion rules are also completely determined by the above formula, and we have

$$
\left[x^{2}\right]=[1]+[x],[y \bar{y}]=[1]+[x],\left[z^{3}\right]=[1],[y]=[x z] .
$$

By (1) of Prop. 4.2, the above formula determines the structure of the ring generated by the coset sectors.

Conj. 2. of 2.3 is also easily verified in this case. For more such "Maverick" cosets, see [DJ2].

\section{REFERENCES}

[ABI] D. Altschuler, M. Bauer and C. Itzykson, The branching rules of conformal embeddings, Comm. Math. Phys., 132, 349-364 (1990).

[B] R. Borcherds, Vertex algebras, Kac-Moody algebras and the Monster, Proc. Nat. Acad. Sci., U.S.A. 83, 3068-3071 (1986).

[BBSS] F.A. Bais, P. Bouwknegt, K. Schoutens, M. Surridge, Coset construction for extended Virasoro algebras, Nucl. Phys. B 304, 371-391, (1988).

[D] C. Dong, Introduction to vertex operator algebras I, Sūrikaisekikenkyūsho Kōkyūroku, No. 904 (1995), 1-25. Also see q-alg/9504017.

[DL] C. Dong and J. Lepowsky, Generalized vertex algebras and relative vertex operators, Progress in Mathematics, 112 (1993).

[DJ1] D. Dunbar and K. Joshi, Characters for Coset conformal field theories and Maverick examples, Inter. J. Mod. Phys. A, Vol.8, No. 23 (1993), 41034121. 
[DJ2] D. Dunbar and K. Joshi, Maverick examples of Coset conformal field theories, Mod. Phys. Letters A, Vol.8, No. 29 (1993), 2803-2814.

[Dix] J. Dixmier, von Neumann Algebras, North-Holland Publishing Company, 1981.

[FG] J. Fröhlich and F. Gabbiani, Operator algebras and Conformal field theory, Comm. Math. Phys., 155, 569-640 (1993).

[FKW] E. Frenkel, V. Kac and M. Wakimoto, Characters and Fusion rules for $W$ algebras via Quantized Drinfeld-Sokolov Reductions, Comm. Math. Phys., 147, 295-328 (1992).

[FLM] I. B. Frenkel, J. Lepowsky and J. Ries, Vertex operator algebras and the Monster, Academic, New York, 1988.

[FZ] I. Frenkel and Y. Zhu, Vertex operator algebras associated to representations of affine and Virasoro algebras, Duke Math. Journal (1992), Vol. 66, No. $1,123-168$.

[GKO] P. Goddard, A. Kent and D. Olive, Unitary representations of Virasoro and super-Virasoro algebras, Comm. Math. Phys. 103 (1986), No. 1, 105-119.

[GL] D. Guido and R. Longo, The conformal spin and statistics theorem, Comm.Math.Phys., 181, 11-35 (1996).

[GW] R. Goodman and N. Wallach, Structure and unitary cocycle representations of loop groups and the group of diffeomorphisms of the circle, J. Reine Angew. Math 347 (1984) 69-133.

[Haag] R. Haag, Local Quantum Physics, Springer-Verlag 1992.

[J] V. Jones, Fusion en alǵebres de Von Neumann et groupes de lacets (d'aprés A. Wassermann), Seminarie Bourbaki, 800, 1-20,1995.

[KW] V. G. Kac and M. Wakimoto, Modular and conformal invariance constraints in representation theory of affine algebras, Advances in Math., 70, 156-234 (1988).

[Kacv] V. G. Kac, Vertex algebras for beginners, AMS, 1997.

[Kac] V. G. Kac, Infinite dimensional Lie algebras, 3rd Edition, Cambridge University Press, 1990.

$[\mathrm{KS}]$ D. Karabali and H. J. Schnitzer, BRST quantization of the gauged WZW action and coset conformal field theories, Nucl. Phys. B 329 (1990), no. 3, 649-666.

[KT] A. Tsuchiya and Y. Kanie, Vertex Operators in conformal field theory on $P^{1}$ and monodromy representations of braid group, Adv. Studies in Pure Math. 16 (88), 297-372.

[L1] R. Longo, Proceedings of International Congress of Mathematicians, 12811291 (1994).

[L2] R. Longo, Duality for Hopf algebras and for subfactors, I, Comm. Math. Phys., 159, 133-150 (1994).

[L3] R. Longo, Index of subfactors and statistics of quantum fields, I, Comm. Math. Phys., 126, 217-247 (1989).

[L4] R. Longo, Index of subfactors and statistics of quantum fields, II, Comm. Math. Phys., 130, 285-309 (1990). 
[L5] R. Longo, An analogue of the Kac-Wakimoto formula and black hole conditional entropy, Comm.Math.Phys., 186, 451-479 (1997).

[L6] R. Longo, Minimal index and braided subfactors, J. Funct.Analysis 109 (1992), 98-112.

[LR] R. Longo and K.-H. Rehren, Nets of subfactors, Rev. Math. Phys., 7, 567-597 (1995).

[Luke] T. Luke, Operator algebras and Conformal Field Theory of the Discrete series representations of Diff $\left(S^{1}\right)$, Dissertation, Cambridge (1994).

[MT] M. Takesaki, Conditional expectation in von Neumann algebra, J. Funct. Analysis 9 (1972), 306-321.

[MS] G. Moore and N. Seiberg, Taming the conformal zoo, Lett. Phys. B , 220, 422-430, (1989).

[Mv] F. J. Murray and J. v. Neumann, On rings of Operators, Ann. Math. 37 (1936), 116-229.

[Po] S. Popa, Classification of amenable subfactors of type II, Acta Math.172 (1994), 352-445.

[PP] M. Pimsner and S. Popa, Entropy and index for subfactors,

Ann. Éc.Norm.Sup. 19, 57-106 (1986).

[PS] A. Pressly and G. Segal, Loop Groups, O.U.P. 1986.

[RS] M. Reed and B. Simon, Methods of Mathematical Physics I: Functional Analysis, Academic Press 1980.

[Stra] S. Stratila, Modular Theory in Operator Algebras, Editura Academiei, 1981.

[SY] A. N. Schellekens and S. Yankielowicz, Field identification fixed points in the coset construction, Nucl. Phys. B 334, 67-102, (1990).

[Wal] M. Walton, Fusion rules of Wess-Zumino-Witten Models, Nuclear physics B, 340: 777-789, 1990.

[We] H.Wenzl, Hecke algebras of type $A$ and subfactors, Invent. Math. 92 (1988), 345-383.

[W1] A. Wassermann, Proceedings of International Congress of Mathematicians, 966-979 (1994).

[W2] A. Wassermann, Operator algebras and Conformal field theories III, Invent. Math. 133 (1998), 467-538.

[Watts] G.M.T. Watts, W-algebras and coset models, Physics Lett.B 245, 65-71, 1990.

[Witten] E. Witten, The central charge in three dimensions, 530-559, V.G. Knizhnik's Memorial Volume, 1989.

[X1] F. Xu, New braided endomorphisms from conformal inclusions, Comm.Math.Phys. 192 (1998) 349-403.

[X2] F. Xu, Applications of braided endomorphisms from conformal inclusions, Inter. Math. Res. Notice., No.1, 5-23 (1998), see also q-alg/9708013, and Erratum, Inter. Math. Res. Notice., No.8, (1998).

[X3] F. Xu, Jones-Wassermann subfactors for disconnected intervals, q-alg/9704003.

[Y] S. Yamagami, A note on Ocneanu's approach to Jones index theory, Inter- 
nat. J. Math., 4, 859-871 (1993).

Department of Mathematics, University of Oklahoma, 601 Elm Ave, Room 423, NORMAN, OK 73019

E-mail address: xufeng@ math.ou.edu 